\title{
Transverse spin in QCD: Radiative corrections
}

\author{
A. Harindranath* and Asmita Mukherjee ${ }^{\dagger}$ \\ Saha Institute of Nuclear Physics, 1/AF, Bidhan Nagar, Calcutta 700064, India \\ Raghunath Ratabole \\ Centre for Theoretical Studies, Indian Institute of Science, Bangalore 560012, India
}

\begin{abstract}
In this paper we address various issues connected with transverse spin in light front QCD. The transverse spin operators, in the $A^{+}=0$ gauge, expressed in terms of the dynamical variables are explicitly interaction dependent unlike the helicity operator which is interaction independent in the topologically trivial sector of light-front QCD. Although it cannot be separated into an orbital and a spin part, we have shown that there exists an interesting decomposition of the transverse spin operator. We discuss the physical relevance of such a decomposition. We perform a one loop renormalization of the full transverse spin operator in light-front Hamiltonian perturbation theory for a dressed quark state. We explicitly show that all the terms dependent on the center of mass momenta get canceled in the matrix element. The entire nonvanishing contribution comes from the fermion intrinsiclike part of the transverse spin operator as a result of cancellation between the gluonic intrinsiclike and the orbital-like part of the transverse spin operator. We compare and contrast the calculations of transverse spin and helicity of a dressed quark in perturbation theory.
\end{abstract}

\section{INTRODUCTION}

From the early days of quantum field theory, it has been recognized that the issues associated with the spin of a composite system in an arbitrary frame are highly complex and non-trivial [1]. In equal-time quantization, the problems arise because of the fact that the Pauli-Lubanski operators, starting from which one can construct the spin operators in a moving frame, are interaction dependent for a composite object. Further, it is quite difficult to separate the center of mass and internal variables which is mandatory in the calculation of spin. Because of these difficulties, there has been rarely any attempt to study the spin of a moving composite system in the conventional equal time formulation of even simple field theoretic models, let alone quantum chromodynamics (QCD).

It is well known that in light-front field theory, in addition to the Hamiltonian, two other operators that belong to the Poincaré group, namely, $F^{i}(i=1,2)$, are interaction dependent. This implies interaction dependent spin operators and this complication is generally thought to be a penalty one has to pay for working with light-front dynamics. In contrast, the angular momentum operators in the familiar instant form of field theory are interaction independent. It is interesting to investigate whether one can understand better the physical origin of the interaction dependence in the light-front case.

A second problem is that, together with the light-front helicity $\mathcal{J}^{3}, F^{i}$ do not obey $S U(2)$ algebra, the commutation relations obeyed by the spin operators of a massive particle. They obey $E(2)$ algebra, appropriate for a massless

\footnotetext{
*Email address: hari@tnp.saha.ernet.in

†Email address: asmita@tnp.saha.ernet.in

${ }^{\ddagger}$ Email address: raghu@cts.iisc.ernet.in
}

particle. This implies that even though $F^{i}$ performs "rotations" about the transverse axes, they have continuous spectrum. It is, however, known how to solve this problem. In terms of the rest of the Poincare generators, one knows [2] how to construct spin operators $\mathcal{J}^{i}$ that together with the helicity $\mathcal{J}^{3}$ obey the $S U(2)$ algebra. One observes that $\mathcal{J}^{i}$ is interaction dependent and has a highly nontrivial operator structure in contrast with $\mathcal{J}^{3}$. Further, unlike $\mathcal{J}^{3}, \mathcal{J}^{i}$ cannot be separated into orbital and spin parts. So far, most of the studies of the transverse spin operators in light-front field theory are restricted to free field theory [3]. Even in this case, the operators have a highly complicated structure. However, one can write these operators as a sum of orbital and spin parts, which can be achieved via a unitary transformation, called Melosh transformation [4]. In interacting theory, presumably this can be achieved order by order in a suitable expansion parameter [5] which is justifiable only in a weakly coupled theory.

Knowledge about transverse rotation operators and transverse spin operators is mandatory for addressing issues concerning Lorentz invariance in light-front theory. Unfortunately, very little is known [6] regarding the field theoretic aspects of the interaction dependent spin operators. We emphasize that in a moving frame, the spin operators are interaction dependent irrespective of whether one considers equal-time field theory or light-front field theory. To the best of our knowledge, in gauge field theory, the canonical structure of spin operators of a composite system in an arbitrary frame has never been studied.

Recently it was shown that [7], starting from the manifestly gauge invariant symmetric energy momentum tensor, in light-front QCD (the gauge $A^{+}=0$ and light-front variables), after the elimination of constrained variables, $\mathcal{J}^{3}$ becomes explicitly interaction independent and can be separated into quark and gluon orbital and spin operators. Thus 
one can write down a helicity sum rule which has a clear physical meaning. The orbital and intrinsic parts of the lightfront helicity operator have also been analyzed recently in [8]. Even though $\mathcal{J}^{i}$ cannot be separated into orbital and spin parts and they are interaction dependent, one can still ask whether one can identify distinct operator structures in $\mathcal{J}^{i}$ and whether one can propose a physically interesting decomposition. Is this decomposition protected by radiative corrections? If distinct operators indeed emerge, do they have any phenomenological consequences especially in deep inelastic scattering which is a light cone dominated process?

Another important issue concerns renormalization. In light-front QCD Hamiltonian, quark mass appears as $m^{2}$ and $m$ terms, $m^{2}$ in the free helicity non-flip part of the Hamiltonian and $m$ in the interaction dependent helicity flip part of the Hamiltonian. It is known that $m^{2}$ and $m$ renormalize differently. $m^{2}$ and $m$ also appear in $\mathcal{J}^{i}$. Do they undergo renormalization? Since $\mathcal{J}^{i}$ are interaction dependent, do they require new counterterms in addition to those necessary to renormalize the Hamiltonian?

In order to resolve the above mentioned problems and puzzles, we have undertaken an investigation of the spin of a composite system in an arbitrary reference frame in QCD. We have compared and contrasted both the instant form and front form formulations. In instant form, even though the angular momentum operators are interaction independent, they qualify as spin operators only in the rest frame of the system. In an arbitrary reference frame, the appropriate spin operators involve, in addition to angular momentum operators, also interaction dependent boost operators. Thus one puzzle is resolved, namely, the interaction dependence of the spin of a composite system in an arbitrary reference frame is not a peculiarity of light-front dynamics, it is a general feature in any formulation of quantum field theory. What is peculiar to light-front dynamics is that one can at most go only to the transverse rest frame of the particle. No frame exists in which $P^{+}=0$ and one is so to speak "always in a moving frame." As a consequence, spin measured in any direction other than that of $P^{+}$cannot be separated into orbital and intrinsic parts. This is to be contrasted with the light-front helicity $\mathcal{J}^{3}$ which is independent of interactions and further can be separated in to orbital and intrinsic parts. The situation is quite analogous to that of a light-like particle. In this case it is well known that since there is no rest frame, one can uniquely identify the spin of the particle only along the direction of motion since only along this direction one can disentangle rotation from translation for a massless particle. Also, in any direction other than the direction of motion, one cannot separate the angular momentum into orbital and intrinsic parts.

In our earlier paper [9], we have shown that even though $\mathcal{J}^{i}$ cannot be separated into orbital and intrinsic parts, one can still achieve a separation into three distinct operator structures. Specifically, starting from the manifestly gauge invariant symmetric energy momentum tensor in QCD, we have derived expressions for the interaction dependent transverse spin operators $\mathcal{J}^{i}(i=1,2)$ which are responsible for the helicity flip of the nucleon in light-front quantization. In order to construct $\mathcal{J}^{i}$, first we have derived expressions for the transverse rotation operators $F^{i}$. In the gauge $A^{+}=0$, we eliminated the constrained variables. In the completely gauge fixed sector, in terms of the dynamical variables, we have shown that one can decompose $\mathcal{J}^{i}=\mathcal{J}_{I}^{i}+\mathcal{J}_{I I}^{i}+\mathcal{J}_{I I I}^{i}$ where only $\mathcal{J}_{I}^{i}$ has explicit coordinate $\left(x^{-}, x^{i}\right)$ dependence in its integrand. The operators $\mathcal{J}_{I I}^{i}$ and $\mathcal{J}_{I I I}^{i}$ arise from the fermionic and bosonic parts respectively of the gauge invariant energy momentum tensor. $\mathcal{J}_{I}^{i}$ is orbital-like and $\mathcal{J}_{I I}^{i}$ and $\mathcal{J}_{I I I}^{i}$ are fermion intrinsic-like and gluon intrinsic-like spin operators respectively.

In this work, we explore the theoretical consequences of the decomposition of $\mathcal{J}^{i}$. We compare and contrast the consequences of this decomposition and the corresponding decomposition of the helicity operator into orbital and spin parts. Next we address the issue of radiative corrections by carrying out the calculation of the transverse spin of a dressed quark in perturbative QCD (PQCD) in the oldfashioned Hamiltonian formalism. To the best of our knowledge, this is for the first time that such a calculation has been performed in quantum field theory. This calculation is facilitated by the fact that boost is kinematical in the light-front formalism. Thus we are able to isolate the internal motion which is only physically relevant from the spurious center of mass motion. We carry out the calculations in a reference frame with arbitrary transverse momentum $P^{\perp}$ and explicitly verify the frame independence of our results. We find that because of cancellation between various interaction independent and dependent operator matrix elements, only one counterterm is needed. We establish the fact the mass counterterm for the renormalization of $\mathcal{J}^{i}$ is the same mass counterterm required for the linear mass term appearing in the interaction dependent helicity flip vertex in QCD. It is important to mention that the divergence structure and renormalization in light-front theory is entirely different from the usual equaltime theory. If one uses constituent momentum cutoff, one violates boost invariance and also encounters non-analytic behavior in the structure of counterterms [10]. In this paper, we have done one loop renormalization of the transverse spin operators by imposing cutoff on the relative transverse momenta and on the longitudinal momentum fraction. Up to one loop, we find that all infrared divergences (in the longitudinal momentum fraction) get canceled in the result. The renormalization of these operators using similarity renormalization technique [10] is to be done in future.

The plan of the paper is as follows. In Sec. II, first, we briefly review the complexities associated with the description of the spin of a composite system in a moving frame in the conventional equal time quantization. Then we give the explicit form of transverse rotation operators in light-front QCD. In Sec. III, we discuss the physical relevance of the decomposition of the transverse spin operator and also compare and contrast it with the helicity operator. In Sec. IV, we present the calculation of the transverse spin for a dressed quark state up to $O\left(\alpha_{s}\right)$ in perturbation theory. Discussion and conclusions are given in Sec. V. The explicit forms of the kinematical operators and the Hamiltonian in light-front QCD starting from the gauge invariant symmetric interaction dependent energy momentum tensor are derived in Appendix 
A. The evaluation of the transverse spin of a system of two free fermions is given in Appendix B. The detailed derivation of the transverse rotation operators in QCD, which are needed for the construction of the transverse spin operators, is given in Appendix C. The full evaluation of the transverse spin operator for a dressed quark in an arbitrary reference frame is given in Appendix D. There we also show the manifest cancellation of all the center of mass momentum dependent terms. Some details of the calculation are provided in Appendix E.

\section{THE TRANSVERSE SPIN OPERATORS IN QCD}

In this section we first discuss the complexities associated with the spin operators for a composite system in equal-time formulation and also compare with the light-front case. Then we give the expressions for interaction dependent transverse rotation operators in light-front QCD starting from the manifestly gauge invariant energy momentum tensor.

The angular momentum density

$$
\mathcal{M}^{\alpha \mu \nu}=x^{\mu} \Theta^{\alpha \nu}-x^{\nu} \Theta^{\alpha \mu} .
$$

In equal time theory, generalized angular momentum

$$
M^{\mu \nu}=\int d^{3} x \mathcal{M}^{0 \mu \nu} .
$$

The rotation operators are $J^{i}=\epsilon^{i j k} M^{j k}$. Thus in a non-gauge theory, all the three components of the rotation operators are manifestly interaction independent. However, the spin operators $S^{i}$ for a composite system in a moving frame involves, in addition to $J^{i}$, the boost operators $K^{i}=M^{0 i}$ which are interaction dependent. Intrinsic spin operators in an arbitrary reference frame in equal-time quantization are given [11] in terms of the Poincaré generators by,

$$
\begin{aligned}
\mathbf{S} & =\frac{1}{M}\left[\mathbf{W}-\frac{\mathbf{P} W^{0}}{M+H}\right] \\
& =\mathbf{J} \frac{P^{0}}{M}-\mathbf{K} \times \frac{\mathbf{P}}{M}-\frac{(\mathbf{J} \cdot \mathbf{P})}{M+P^{0}} \frac{\mathbf{P}}{M}
\end{aligned}
$$

where $\mathbf{W}$ are the space components of the Pauli-Lubanski operator, $W^{\mu}=-\frac{1}{2} \epsilon^{\mu \nu \rho \lambda} M_{\nu \rho} P_{\lambda} . H, \vec{P}$ are equal time Hamiltonian and momentum operators respectively obtained by integrating the energy momentum tensor over a spacelike surface and $\vec{J}$ and $\vec{K}$ are the equal time rotation and boost generators respectively, which are obtained by integrating the angular momentum density over a spacelike surface. Since boost $\mathbf{K}$ is dynamical, all the three components of $\mathbf{S}$ are interaction dependent in the equal time quantization. Nevertheless, the component of $\mathbf{S}$ along $\mathbf{P}$ remains kinematical. This is to be compared with light-front quantization where the third component of the light-front spin operator $\mathcal{J}^{3}$ is kinematical. This arises from the facts that boost operators are kinematical on the light front, the interaction dependence of light-front spin operators $\mathcal{J}^{i}$ arises solely from the rotation operators, and the third component of the rotation operator $J^{3}$ is kinematical on the light front.
A further complication arises in equal time quantization. In order to describe the intrinsic spin of a composite system, one should be able to separate the center of mass motion from the internal motion. Even in free field theory, this turns out to be quite involved (see Ref. [12] and references therein). On the other hand, in light-front theory, since transverse boosts are simply Galilean boosts, separation of center of mass motion and internal motion is as simple as in nonrelativistic theory (see Appendix D).

A gauge invariant separation of the nucleon angular momentum is performed in Ref. [13]. However, as far the spin operator in an arbitrary reference frame is concerned, the analysis of this reference is valid only in the rest frame where spin coincides with total angular momentum operator. Further, there is no mention of the complications in the equal time theory, which arise from the need to project out the center of mass motion in an arbitrary reference frame. Moreover, the distinction between the longitudinal and transverse components of the spin is not made. It is crucial to make this distinction since physically the longitudinal and transverse components of the spin carry quite distinct information (as is clear, for example, from the spin of a massless particle). Moreover, even for the third component of the spin of a composite system in a moving frame, there is crucial difference between equal time and light front cases. $\mathcal{J}^{3}$ (helicity) is interaction independent whereas $S^{3}$ is interaction dependent in general except when measured along the direction of P.

In light-front theory, generalized angular momentum

$$
M^{\mu \nu}=\frac{1}{2} \int d x^{-} d^{2} x^{\perp} \mathcal{M}^{+\mu \nu} .
$$

$J^{3}$ which is related to the helicity is given by

$$
J^{3}=M^{12}=\frac{1}{2} \int d x^{1} d^{2} x^{\perp}\left[x^{1} \Theta^{+2}-x^{2} \Theta^{+1}\right]
$$

and is interaction independent. On the other hand, the transverse rotation operators which are related to the transverse spin are given by

$$
F^{i}=M^{-i}=\frac{1}{2} \int d x^{-} d^{2} x^{\perp}\left[x^{-} \Theta^{+i}-x^{i} \Theta^{+-}\right] .
$$

They are interaction dependent even in a non-gauge theory since $\Theta^{+-}$is the Hamiltonian density.

For a massive particle, the transverse spin operators [2] $\mathcal{J}^{i}$ in light-front theory are given in terms of Poincaré generators by

$$
\begin{aligned}
& M \mathcal{J}^{1}=W^{1}-P^{1} \mathcal{J}^{3}=\frac{1}{2} F^{2} P^{+}+K^{3} P^{2}-\frac{1}{2} E^{2} P^{-}-P^{1} \mathcal{J}^{3} \\
& M \mathcal{J}^{2}=W^{2}-P^{2} \mathcal{J}^{3}=-\frac{1}{2} F^{1} P^{+}-K^{3} P^{1}+\frac{1}{2} E^{1} P^{-}-P^{2} \mathcal{J}^{3}
\end{aligned}
$$


The first term in Eqs. (2.6) and (2.7) contains both center of mass motion and internal motion and the next three terms in these equations serve to remove the center of mass motion.

The helicity operator is given by

$$
\mathcal{J}^{3}=\frac{W^{+}}{P^{+}}=J^{3}+\frac{1}{P^{+}}\left(E^{1} P^{2}-E^{2} P^{1}\right) .
$$

Here, $J^{3}$ contain both center of mass motion and internal motion and the other two terms serve to remove the center of mass motion. The operators $\mathcal{J}^{i}$ obey the angular momentum commutation relations

$$
\left[\mathcal{J}^{i}, \mathcal{J}^{j}\right]=i \epsilon^{i j k} \mathcal{J}^{k} .
$$

In order to calculate the transverse spin operators, first we need to construct the Poincare generators $P^{+}, P^{i}, P^{-}, K^{3}, E^{i}, J^{3}$ and $F^{i}$ in light-front QCD. The explicit form of the operator $J^{3}$ is given Ref. [7]. The con- struction of $F^{i}$ which is algebraically quite involved is carried out in Appendix C. The final form of $F^{i}$ is also given in Ref. [9]. The construction of the rest of the kinematical operators is given in Appendix A. In this Appendix we have presented all the operators in the manifestly Hermitian form, which is necessary, as we shall see later.

In order to have a physical picture of the complicated situation at hand, it is instructive to calculate the spin operator in free field theory. The case of two free massive fermions is carried out in Appendix B.

In light-front theory we set the gauge $A^{+}=0$ and eliminate the dependent variables $\psi^{-}$and $A^{-}$using the equations of constraint. We have shown that [9] (for details of the derivation see Appendix C), in the topologically trivial sector of the theory one can write the transverse rotation operator as

$$
F^{2}=F_{I}^{2}+F_{I I}^{2}+F_{I I I}^{2}
$$

where

$$
\begin{aligned}
F_{I}^{2}= & \frac{1}{2} \int d x^{-} d^{2} x^{\perp}\left[x^{-} \mathcal{P}_{0}^{2}-x^{2}\left(\mathcal{H}_{0}+\mathcal{V}\right)\right] \\
F_{I I}^{2}= & \frac{1}{2} \int d x^{-} d^{2} x^{\perp}\left[\xi^{\dagger}\left[\sigma^{3} \partial^{1}+i \partial^{2}\right] \frac{1}{\partial^{+}} \xi+\left[\frac{1}{\partial^{+}}\left(\partial^{1} \xi^{\dagger} \sigma^{3}-i \partial^{2} \xi^{\dagger}\right)\right] \xi\right]+\frac{1}{2} \int d x^{-} d^{2} x^{\perp} m\left[\xi^{\dagger}\left[\frac{\sigma^{1}}{i \partial^{+}} \xi\right]-\left[\frac{1}{i \partial^{+}} \xi^{\dagger} \sigma^{1}\right] \xi\right] \\
& +\frac{1}{2} \int d x^{-} d^{2} x^{\perp} g\left[\xi^{\dagger} \frac{1}{\partial^{+}}\left[\left(-i \sigma^{3} A^{1}+A^{2}\right) \xi\right]+\frac{1}{\partial^{+}}\left[\xi^{\dagger}\left(i \sigma^{3} A^{1}+A^{2}\right)\right] \xi\right] \\
F_{I I I}^{2}= & -\int d x^{-} d^{2} x^{\perp} 2\left(\partial^{1} A^{1}\right) A^{2}-\frac{1}{2} \int d x^{-} d^{2} x^{\perp} g \frac{4}{\partial^{+}}\left(\xi^{\dagger} T^{a} \xi\right) A^{2 a}-\frac{1}{2} \int d x^{-} d^{2} x^{\perp} g f^{a b c} \frac{2}{\partial^{+}}\left(A^{i b} \partial^{+} A^{i c}\right) A^{2 a}
\end{aligned}
$$

Here $\mathcal{P}_{0}^{i}$ is the free momentum density, $\mathcal{H}_{o}$ is the free Hamiltonian density and $\mathcal{V}$ are the interaction terms in the Hamiltonian in manifestly Hermitian form (see Appendix A). The operators $F_{I I}^{2}$ and $F_{I I I}^{2}$ whose integrands do not explicitly depend upon coordinates arise from the fermionic and bosonic parts respectively of the gauge invariant symmetric energy momentum tensor in QCD. The above separation is slightly different from that in [9]. From Eq. (2.6) in Sec. II it follows that the transverse spin operators $\mathcal{J}^{i}$, $(i$ $=1,2$ ) can also be written as the sum of three parts, $\mathcal{J}_{I}^{i}$ whose integrand has explicit coordinate dependence, $\mathcal{J}_{I I}^{i}$ which arises from the fermionic part, and $\mathcal{J}_{I I I}^{i}$ which arises from the bosonic part of the energy momentum tensor.

In the next section, we propose a decomposition of transverse spin in analogy with the helicity case and compare and contrast the two cases.

\section{THE DECOMPOSITION OF TRANSVERSE SPIN}

The transverse spin operators $\mathcal{J}^{i}$ in light-front theory for a massive particle can be given in terms of Poincaré generators by Eq. (2.6). In [7] it has been shown explicitly that the helicity operator $\mathcal{J}^{3}$ in the light-front gauge, in terms of the dynamical fields in the topologically trivial sector of QCD can be written as

$$
\mathcal{J}^{3}=\mathcal{J}_{f i}^{3}+\mathcal{J}_{f o}^{3}+\mathcal{J}_{g o}^{3}+\mathcal{J}_{g i}^{3}
$$

where $\mathcal{J}_{f i}^{3}$ is the fermion intrinsic part, $\mathcal{J}_{\text {fo }}^{3}$ is the fermion orbital part, $\mathcal{J}_{\text {go }}^{3}$ is the gluon orbital part and $\mathcal{J}_{g i}^{3}$ is the gluon intrinsic part. The helicity sum rule is given by, for a longitudinally polarized fermion state,

$$
\frac{1}{\mathcal{N}}\left\langle P S^{\|}\left|\mathcal{J}_{f i}^{3}+\mathcal{J}_{f o}^{3}+\mathcal{J}_{g o}^{3}+\mathcal{J}_{g i}^{3}\right| P S^{\|}\right\rangle= \pm \frac{1}{2}
$$

In the transverse rest frame $\left(P^{\perp}=0\right)$, the helicity sum rule takes the form

$$
\frac{1}{\mathcal{N}}\left\langle P S^{\|}\left|J_{f i}^{3}+J_{f o}^{3}+J_{g o}^{3}+J_{g i}^{3}\right| P S^{\|}\right\rangle= \pm \frac{1}{2}
$$


For a boson state, RHS of the above equation should be replaced with the corresponding helicity. Here, $\mathcal{N}$ is the normalization constant of the state. Unlike the helicity operator, which can be separated into orbital and spin parts, the transverse spin operators cannot be written as a sum of orbital and spin contributions. Only in the free theory, one can write them as a sum of orbital and spin parts by a unitary transformation called Melosh transformation. However, we have shown that they can be separated into three distinct components. At this point, we would also like to contrast our work with Ref. [13], where a gauge invariant decomposition of nucleon spin has been done. The analysis in Ref. [13] has been performed in the rest frame of the hadron and no distinction is made between helicity and transverse spin, whereas, we have worked in the gauge fixed theory in an arbitrary reference frame.

In analogy with the helicity sum rule, we propose a decomposition of the transverse spin, which can be written as

$$
\frac{1}{\mathcal{N}}\left\langle P S^{\perp}\left|\mathcal{J}_{I}^{i}+\mathcal{J}_{I I}^{i}+\mathcal{J}_{I I I}^{i}\right| P S^{\perp}\right\rangle= \pm \frac{1}{2}
$$

for a fermion state polarized in the transverse direction. For a bosonic state, RHS will be replaced with the corresponding transverse component of spin.

What is the physical relevance of such a decomposition of the transverse spin operator? The fermion intrinsic part of the helicity operator can be related to the first moment of the quark helicity distribution measured in longitudinally polarized deep inelastic scattering. In the case of the transverse spin operator, we have shown [9] that there exists a direct connection between the hadron expectation value of the fermionic intrinsic-like part of the transverse spin operator $\mathcal{J}_{I I}^{i}$ and the integral of the quark distribution function $g_{T}$ that appear in transversely polarized deep inelastic scattering. Also we can identify [9] the operators that are present in the hadron expectation value of $\mathcal{J}_{I I I}^{i}$ with the operator structures that are present in the integral of the gluon distribution function that appear in transverse polarized hard scattering. The physical relevance of the decomposition is made clear from the identification. Our results show the intimate connection between transverse spin in light-front QCD and transverse polarized deep inelastic scattering. As far as we know, such connections are not established so far in instant form of field theory and this is the first time that the first moment of $g_{T}$ is related to a conserved quantity. It is already known that the interaction independent light-front helicity operator $\mathcal{J}^{3}$ can be separated as $\mathcal{J}^{3}=\mathcal{J}_{q(i)}^{3}+\mathcal{J}_{q(o)}^{3}+\mathcal{J}_{g(i)}^{3}+\mathcal{J}_{g(o)}^{3}$ and further, hadron expectation value of $\mathcal{J}_{q(i)}^{3}$ is directly related to the integral of the deep inelastic helicity structure function $g_{1}$. Thus we find natural physical explanation for the simplicity and complexity of operator structures appearing in the structure functions $g_{1}$ and $g_{T}$ respectively. Another important point is that in perturbation theory, the helicity flip interactions which are proportional to mass play a crucial role both in $g_{T}$ and in the transverse spin operator whereas they are not important in the case of the helicity operator.

Because the transverse spin operators are interaction dependent, they acquire divergences in perturbation theory.
One has to regularize them by imposing momentum cutoffs and in the regularized theory the Poincare algebra as well as the commutation relation obeyed by the spin operators are violated [14]. One has to introduce appropriate counterterms to restore the algebra. In the next section, we perform the renormalization of the full transverse spin operator up to $O\left(\alpha_{s}\right)$ in light-front Hamiltonian perturbation theory by evaluating the matrix element for a quark state dressed with one gluon. This calculation also verifies the relation (3.4) up to $O\left(\alpha_{s}\right)$ in perturbation theory.

\section{TRANSVERSE SPIN OF A DRESSED QUARK IN PERTURBATION THEORY}

In this section, we evaluate the expectation value of the transverse spin operator in perturbative QCD for a dressed quark state.

The dressed quark state with fixed helicity $\sigma$ can be expanded in Fock space as

$$
\begin{aligned}
|P, \sigma\rangle= & \phi_{1}^{\lambda} b^{\dagger}(P, \sigma)|0\rangle \\
& +\sum_{\sigma_{1}, \lambda_{2}} \int \frac{d k_{1}^{+} d^{2} k_{1}^{\perp}}{\sqrt{2(2 \pi)^{3} k_{1}^{+}}} \int \frac{d k_{2}^{+} d^{2} k_{2}^{\perp}}{\sqrt{2(2 \pi)^{3} k_{2}^{+}}} \\
& \times \sqrt{2(2 \pi)^{3} P^{+}} \delta^{3}\left(P-k_{1}-k_{2}\right) \\
& \times \phi_{\sigma_{1}, \lambda_{2}}^{\sigma}\left(P, \mid k_{1}, ; k_{2}\right) b^{\dagger}\left(k_{1}, \sigma_{1}\right) a^{\dagger}\left(k_{2}, \lambda_{2}\right)|0\rangle .
\end{aligned}
$$

We are considering dressing with one gluon since we shall evaluate the expectation value up to $O\left(g^{2}\right)$. The normalization of the state is given by

$$
\left\langle k^{\prime}, \lambda^{\prime} \mid k, \lambda\right\rangle=2(2 \pi)^{3} k^{+} \delta_{\lambda \lambda^{\prime}} \delta\left(k^{+}-k^{\prime+}\right) \delta\left(k^{\perp}-k^{\prime \perp}\right) .
$$

The quark target transversely polarized in the $x$ direction can be expressed in terms of helicity up and down states by

$$
\left|k^{+}, k^{\perp}, s^{1}\right\rangle=\frac{1}{\sqrt{2}}\left(\left|k^{+}, k^{\perp}, \uparrow\right\rangle \pm\left|k^{+}, k^{\perp}, \downarrow\right\rangle\right)
$$

with $s^{1}= \pm m_{R}$, where $m_{R}$ is the renormalized mass of the quark.

We introduce the boost invariant amplitudes $\Phi_{1}^{\lambda}$ and $\Phi_{\sigma_{1} \lambda_{2}}^{\lambda}\left(x, q^{\perp}\right) \quad$ respectively by $\phi^{\lambda}(k)=\Phi_{1}^{\lambda} \quad$ and $\phi_{\lambda_{1} \lambda_{2}}^{\lambda}\left(k ; k_{1}, k_{2}\right)=\left(1 / \sqrt{k^{+}}\right) \Phi_{\lambda_{1} \lambda_{2}}^{\lambda}\left(x, q^{\perp}\right)$, where $x=k_{1}^{+} / P^{+}$ and $q^{\perp}=k_{1}^{\perp}-x P^{\perp}$. From the light-front QCD Hamiltonian, to lowest order in perturbative QCD, we have 


$$
\begin{aligned}
\Phi_{\sigma_{1}, \sigma_{2}}^{\lambda}\left(x, q^{\perp}\right)= & -\frac{x(1-x)}{\left(q^{\perp}\right)^{2}+m^{2}(1-x)^{2}} \frac{1}{\sqrt{1-x}} \\
& \times \frac{g}{\sqrt{2(2 \pi)^{3}}} T^{a} \chi_{\sigma_{1}}^{\dagger}\left[2 \frac{q^{\perp}}{1-x}+\frac{\tilde{\sigma}^{\perp} \cdot q^{\perp}}{x} \widetilde{\sigma}^{\perp}\right. \\
& \left.-\tilde{\sigma}^{\perp} i m \frac{1-x}{x}\right] \chi_{\lambda} \cdot\left(\epsilon_{\sigma_{2}}^{\perp}\right)^{*} \Phi_{1}^{\lambda} .
\end{aligned}
$$

Here $m$ is the quark mass and $x$ is the longitudinal momentum carried by the quark. Also, $\tilde{\sigma}^{1}=\sigma^{2}$ and $\tilde{\sigma}^{2}=-\sigma^{1}$. It is to be noted that the $m$ dependence in the above wave function arises from the helicity flip part of the light-front QCD Hamiltonian. This term plays a very important role in the case of transversely polarized target states.

For simplicity, in this section, we calculate the matrix element of the transverse spin operator for a dressed quark state in a frame where the transverse momentum of the quark is zero. It can be seen from Eq. (2.6) that the sole contribution in this case comes from the first term in the RHS, namely the transverse rotation operator. A detailed calculation of the matrix elements of the transverse spin operator in an arbitrary reference frame is given in Appendix D where we have explicitly shown that all the terms depending on $P^{\perp}$ get canceled.

The matrix elements presented below have been evaluated between states of different helicities, namely $\sigma$ and $\sigma^{\prime}$. Since the transversely polarized state can be expressed in terms of the longitudinally polarized (helicity) states by Eq. (4.3), the matrix elements of these operators between transversely polarized states can be easily obtained from these expressions.

Here, we have used the manifest Hermitian form of all the operators. It is necessary to keep manifest Hermiticity at each intermediate step to cancel terms containing derivative of delta function. [15],

The operator $\frac{1}{2} F^{2} P^{+}$can be separated into three parts

$$
\frac{1}{2} F^{2} P^{+}=\frac{1}{2} F_{I}^{2} P^{+}+\frac{1}{2} F_{I I}^{2} P^{+}+\frac{1}{2} F_{I I I}^{2} P^{+},
$$

where $F_{I}^{2}, F_{I I}^{2}$ and $F_{I I I}^{2}$ have been defined earlier. The matrix elements of the different parts of these for a dressed quark state are given below. The evaluation of the matrix element of $\frac{1}{2} F_{I}^{2} P^{+}$is quite complicated since it involves derivatives of delta functions. A part of this calculation has been given in some detail in Appendix E. The operator

$$
\frac{1}{2} F_{I}^{2} P^{+}=\frac{1}{2} F_{I}^{2}(1) P^{+}-\frac{1}{2} F_{I}^{2}(2) P^{+}-\frac{1}{2} F_{I}^{2}(3) P^{+} .
$$

The first term contains the momentum density, the second and the third terms contain the free and the interaction parts of the Hamiltonian density respectively. The matrix elements are given by

$$
\begin{aligned}
\langle P, \sigma| \frac{1}{2} & F_{I}^{2}(1) P^{+}\left|P, \sigma^{\prime}\right\rangle \\
& =\left\langle P, \sigma\left|\frac{1}{2} \int d x d^{2} q^{\perp} x^{-} P_{0}^{2} \frac{1}{2} P^{+}\right| P, \sigma^{\prime}\right\rangle \\
& =-\frac{i}{2} \sum_{\text {spin }} \int d x d^{2} q^{\perp} q^{2} \Phi_{\sigma_{1}^{\lambda}}^{* \sigma} \frac{\partial \Phi_{\sigma_{1}^{\prime} \lambda^{\prime}}^{* \sigma^{\prime}}}{\partial x}+\text { H.c. }
\end{aligned}
$$

$$
\begin{aligned}
\langle P, \sigma| \frac{1}{2} & F_{I}^{2}(2) P^{+}\left|P, \sigma^{\prime}\right\rangle \\
= & \left\langle P, \sigma\left|\frac{1}{2} \int d x d^{2} q^{\perp} x^{2} P_{0}^{-} \frac{1}{2} P^{+}\right| P, \sigma^{\prime}\right\rangle \\
= & \frac{i}{4} \sum_{\text {spin }} \int d x d^{2} q^{\perp} \Phi_{\sigma_{1} \lambda}^{* \sigma} \frac{\partial \Phi_{\sigma_{1}^{\prime} \lambda^{\prime}}^{\sigma^{\prime}}}{\partial q^{2}}\left(q^{\perp}\right)^{2} \\
& \times\left(\frac{1-x}{x}-\frac{x}{1-x}\right)+\frac{i}{4} \sum_{\text {spin }} \int d x d^{2} q^{\perp} m^{2} \\
& \times \frac{1-x}{x} \Phi_{\sigma_{1} \lambda}^{* \sigma} \frac{\partial \Phi_{\sigma_{1}^{\prime} \lambda^{\prime}}^{\sigma^{\prime}}}{\partial q^{2}}+\text { H.c. }
\end{aligned}
$$

In the above two equations, both the single particle and two particle diagonal matrix elements contribute. Here, H.c. is the Hermitian conjugate, $\Sigma_{\text {spin }}$ is the summation over $\sigma_{1}, \sigma_{1}^{\prime}, \lambda_{1}, \lambda_{1}^{\prime} . P_{0}^{-}$is the free part of the Hamiltonian density:

$$
\begin{aligned}
\langle P, \sigma| & \frac{1}{2} F_{I}^{2}(3) P^{+}\left|P, \sigma^{\prime}\right\rangle \\
= & \left\langle P, \sigma\left|\frac{1}{2} \int d x d^{2} q^{\perp} x^{2} P_{\text {int }}^{-} \frac{1}{2} P^{+}\right| P, \sigma^{\prime}\right\rangle \\
= & \frac{g}{\sqrt{2(2 \pi)^{3}}} \sum_{\text {spin }} \int d x d^{2} q^{\perp} \frac{1}{\sqrt{1-x}}\left(-\frac{i}{4} \Phi_{1}^{* \sigma} \chi_{\sigma}^{\dagger}\right. \\
& \left.\times\left[\tilde{\sigma}^{2}\left(\tilde{\sigma}^{\perp} \cdot \epsilon^{\perp}\right)+\frac{\left(\tilde{\sigma}^{\perp} \cdot \epsilon^{\perp}\right) \tilde{\sigma}^{2}}{x}\right] \chi_{\sigma_{1}} \Phi_{\sigma_{1} \lambda}^{\sigma^{\prime}}+\text { H.c. }\right) .
\end{aligned}
$$

$P_{\text {int }}^{-}$is the interaction part of the light-front QCD Hamiltonian density. Only the $q q g$ part of it contributes to the dressed quark matrix element.

The operator $\frac{1}{2} F_{I I}^{2} P^{+}$which originates from the fermionic part of the energy momentum tensor, can be separated into three parts:

$$
\frac{1}{2} F_{I I}^{2} P^{+}=\frac{1}{2} F_{m I I}^{2} P^{+}+\frac{1}{2} F_{q^{\perp} I I}^{2} P^{+}+\frac{1}{2} F_{g I I}^{2} P^{+}
$$


where $\frac{1}{2} F_{m I I}^{2} P^{+}$is the explicit mass dependent part of the operator, $\frac{1}{2} F_{q^{\perp} I I}^{2} P^{+}$is the part containing derivatives with respect to $x^{\perp}$ and $\frac{1}{2} F_{g I I}^{2} P^{+}$is the interaction part. The matrix elements are given by

$$
\begin{aligned}
\langle P, \sigma| & \frac{1}{2} F_{m I I}^{2} P^{+}\left|P, \sigma^{\prime}\right\rangle \\
= & \frac{m}{2} \Phi_{1}^{* \sigma} \Phi_{1}^{\sigma^{\prime}}+\frac{m}{2} \sum_{\text {spin }} \int d x d^{2} q^{\perp} \\
& \times \Phi_{\sigma_{1} \lambda}^{* \sigma} \chi_{\sigma_{1}}^{\dagger} \sigma^{1} \chi_{\sigma_{1}^{\prime}} \Phi_{\sigma_{1}^{\prime} \lambda^{\prime}}^{\sigma^{\prime}} \frac{1}{x}, \\
\langle P, \sigma| & \frac{1}{2} F_{q^{\perp} I I}^{2} P^{+}\left|P, \sigma^{\prime}\right\rangle \\
= & \frac{1}{2} \sum_{s p i n} \int d x d^{2} q^{\perp} \Phi_{\sigma_{1} \lambda}^{* \sigma} \chi_{\sigma_{1}}^{\dagger} \sigma^{3} q^{1} \chi_{\sigma_{1}^{\prime}} \Phi_{\sigma_{1}^{\prime} \lambda^{\prime}}^{\sigma^{\prime}} \frac{1}{x}, \\
\langle P, \sigma| & \frac{1}{2} F_{g I I}^{2} P^{+}\left|P, \sigma^{\prime}\right\rangle \\
= & \frac{1}{4} \frac{g}{\sqrt{2(2 \pi)^{3}}} \sum_{s p i n} \int d x d^{2} q^{\perp} \frac{1}{\sqrt{1-x}} \\
& \times\left(i \Phi _ { 1 } ^ { * \sigma } \left[\chi_{\sigma}^{\dagger}\left(-i \sigma^{3} \epsilon_{\lambda}^{1}+\epsilon_{\lambda}^{2}\right) \chi_{\sigma_{1}}\right.\right. \\
& \left.\left.-\frac{1}{x} \chi_{\sigma}^{\dagger}\left(i \sigma^{3} \epsilon_{\lambda}^{1}+\epsilon_{\lambda}^{2}\right) \chi_{\sigma_{1}}\right] \Phi_{\sigma_{1} \lambda}^{\sigma^{\prime}}+\text { H.c. }\right) .
\end{aligned}
$$

In Eqs. (4.11) and (4.12), contributions come from only diagonal matrix elements whereas Eq. (4.13) contain only offdiagonal matrix elements. The matrix element of $\frac{1}{2} F_{I I I}^{2} P^{+}$, which comes from the gluonic part, is given by

$$
\begin{aligned}
\langle P, \sigma| & \frac{1}{2} F_{I I I}^{2} P^{+}\left|P, \sigma^{\prime}\right\rangle \\
= & -\frac{g}{\sqrt{2(2 \pi)^{3}}} \sum_{\text {spin }} \int d x d^{2} q^{\perp} \frac{1}{\sqrt{1-x}} \\
& \times\left(\Phi_{1}^{* \sigma} \epsilon_{\lambda}^{2} \Phi_{\sigma_{1} \lambda}^{\sigma^{\prime}} \frac{1}{i(1-x)}+\text { H.c. }\right) \\
& -\int d x d^{2} q^{\perp} \frac{q^{1}}{(1-x)} \sum_{\lambda, \sigma_{1}, \sigma_{1}^{\prime}} \lambda \Phi_{\sigma_{1} \lambda}^{* \sigma} \Phi_{\sigma_{1}^{\prime} \lambda}^{\sigma^{\prime}}
\end{aligned}
$$

The first term in the RHS is the off-diagonal contribution which comes from the interaction dependent part of the operator. The second term is the diagonal contribution coming from the free part.

The expectation value of the transverse spin operator between transversely polarized states is given by

$$
\begin{aligned}
\left\langle P, S^{1}\left|M \mathcal{J}^{1}\right| P, S^{1}\right\rangle= & \left\langle P, S^{1}\right| \frac{1}{2} F^{2} P^{+}+K^{3} P^{2} \\
& -\frac{1}{2} E^{2} P^{-}-P^{1} \mathcal{J}^{3}\left|P, S^{1}\right\rangle .
\end{aligned}
$$

Since we are in the reference frame with zero $P^{\perp}$, only the first term in the RHS, i.e. the $\frac{1}{2} F^{2} P^{+}$term will contribute, as mentioned earlier. We substitute for $\Phi_{\sigma_{1} \lambda}^{\sigma}$ using Eq. (4.4). The final forms of the matrix elements are given by

$$
\begin{aligned}
& \left\langle P, S^{1}\left|M \mathcal{J}_{I}^{1}(1)\right| P, S^{1}\right\rangle=-\frac{m \alpha_{s}}{4 \pi} C_{f} \ln \frac{Q^{2}}{\mu^{2}} \int_{\epsilon}^{1-\epsilon} d x(1+x), \\
& \left\langle P, S^{1}\left|M \mathcal{J}_{I}^{1}(2)\right| P, S^{1}\right\rangle=\frac{m \alpha_{s}}{4 \pi} C_{f} \ln \frac{Q^{2}}{\mu^{2}} \int_{\epsilon}^{1-\epsilon} d x(1-2 x), \\
& \left\langle P, S^{1}\left|M \mathcal{J}_{I}^{1}(3)\right| P, S^{1}\right\rangle=-\frac{m \alpha_{s}}{4 \pi} C_{f} \ln \frac{Q^{2}}{\mu^{2}} \int_{\epsilon}^{1-\epsilon} d x(1-x)
\end{aligned}
$$

where $M \mathcal{J}_{I}^{1}(1), M \mathcal{J}_{I}^{1}(2)$ and $M \mathcal{J}_{I}^{1}(3)$ are related respectively to $F_{I}^{2}(1), F_{I}^{2}(2)$ and $F_{I}^{2}(3)$ defined earlier. $\mu$ is the hadronic factorization scale for separating the "hard" and "soft" dynamics of QCD, i.e. we have set a hadronic scale such that $\left|q^{\perp}\right|^{2} \gg \mu^{2} \gg m^{2}$. $\epsilon$ is a small cutoff on the longitudinal momentum fraction.

So we obtain, from the above three expressions, using Eq. (4.6),

$$
\left\langle P, S^{1}\left|M \mathcal{J}_{I}^{1}\right| P, S^{1}\right\rangle=-\frac{m \alpha_{s}}{4 \pi} C_{f} \ln \frac{Q^{2}}{\mu^{2}} .
$$

The contribution to the matrix element of $M \mathcal{J}_{I I}^{1}$ entirely comes from $F_{I I}^{2}$. The various parts of this matrix element are given by

$$
\begin{aligned}
\left\langle P, S^{1}\left|M \mathcal{J}_{m I I}^{1}\right| P, S^{1}\right\rangle= & \frac{1}{2} m\left|\Phi_{1}^{\sigma}\right|^{2} \\
& +\frac{m \alpha_{s}}{2 \pi} C_{f} \ln \frac{Q^{2}}{\mu^{2}} \int_{\epsilon}^{1-\epsilon} d x \frac{1}{1-x}, \\
\left\langle P, S^{1}\left|M \mathcal{J}_{q^{\perp} I I}^{1}\right| P, S^{1}\right\rangle= & -\frac{m \alpha_{s}}{4 \pi} C_{f} \ln \frac{Q^{2}}{\mu^{2}} \int_{\epsilon}^{1-\epsilon} d x(1-x), \\
\left\langle P, S^{1}\left|M \mathcal{J}_{g I I}^{1}\right| P, S^{1}\right\rangle= & \frac{m \alpha_{s}}{4 \pi} C_{f} \ln \frac{Q^{2}}{\mu^{2}} \frac{1}{2},
\end{aligned}
$$

where $M \mathcal{J}_{m I I}^{1}, M \mathcal{J}_{q^{\perp} I I}^{1}$ and $M \mathcal{J}_{g I I}^{1}$ are related respectively to $F_{m I I}^{2}, F_{q^{\perp} I I}^{2}$ and $F_{g I I}^{2}$. In Eq. (4.20) we have to use the normalization condition, 


$$
\left|\Phi_{1}^{\sigma}\right|^{2}=1-\frac{\alpha_{s}}{2 \pi} C_{f} \ln \frac{Q^{2}}{\mu^{2}} \int_{\epsilon}^{1-\epsilon} d x \frac{1+x^{2}}{1-x} .
$$

Up to $O\left(\alpha_{s}\right)$, the normalization condition will contribute only in the first term of Eq. (4.20). We get, from Eq. (4.20),

$$
\begin{aligned}
& \left\langle P, S^{1}\left|M \mathcal{J}_{m I I}^{1}\right| P, S^{1}\right\rangle \\
& \quad=\frac{1}{2} m+\frac{m \alpha_{s}}{4 \pi} C_{f} \ln \frac{Q^{2}}{\mu^{2}} \int_{\epsilon}^{1-\epsilon} d x\left(\frac{2}{1-x}-\frac{1+x^{2}}{1-x}\right) .
\end{aligned}
$$

It is clear that the singularity at $x=1$ is canceled due to the contribution from the normalization condition. The overall contribution coming from $M \mathcal{J}_{I I}^{1}$ is given by

$$
\left\langle P, S^{1}\left|M \mathcal{J}_{I I}^{1}\right| P, S^{1}\right\rangle=\frac{m}{2}\left(1+\frac{3 \alpha_{s}}{4 \pi} C_{f} \ln \frac{Q^{2}}{\mu^{2}}\right),
$$

which does not involve any $x$ divergence. The matrix element of $M \mathcal{J}_{I I I}^{1}$ is given by

$$
\left\langle P, S^{1}\left|M \mathcal{J}_{I I I}^{1}\right| P, S^{1}\right\rangle=\frac{2 m \alpha_{s}}{4 \pi} C_{f} \ln \frac{Q^{2}}{\mu^{2}} \int_{\epsilon}^{1-\epsilon}(1-x) d x .
$$

It is to be noted that all the contributing matrix elements are proportional to the quark mass. Among the different parts of the operator, only $\mathcal{J}_{m I I}^{i}$ and a part of the interaction terms in $\mathcal{J}_{I}^{i}$ [see Eq. (2.11)] are proportional to the quark mass $m$. These mass dependent terms flip the quark helicity. It is also to be noted that the terms proportional to $m^{2}$ do not flip the helicity. In all the other terms, though the operators do not depend on $m$ explicitly, the contributions to the matrix elements arise from the interference of the $m$ terms in the wave function of Eq. (4.4), with the non- $m$ dependent terms through the different parts of the transverse spin operator. Since in light-front formulation, helicity and chirality are the same, these linear in $m$ terms are explicit chiral symmetry breaking terms. From Eq. (4.19) and Eq. (4.26) we find that

$$
\begin{aligned}
& \left\langle P, S^{1}\left|M \mathcal{J}_{I}^{1}+M \mathcal{J}_{I I I}^{1}\right| P, S^{1}\right\rangle \\
& =\frac{m \alpha_{s}}{4 \pi} C_{f} \ln \frac{Q^{2}}{\mu^{2}} \int_{\epsilon}^{1-\epsilon}(1-2 x) d x=0
\end{aligned}
$$

which means that the entire contribution to the matrix element of the transverse spin operator is given by

$$
\left\langle P, S^{1}\left|M \mathcal{J}^{1}\right| P, S^{1}\right\rangle=\frac{m}{2}\left(1+\frac{3 \alpha_{s}}{4 \pi} C_{f} \ln \frac{Q^{2}}{\mu^{2}}\right) .
$$

This contribution entirely comes from $M \mathcal{J}_{I I}^{1}$. Contribution from the orbital-like part $\left(M \mathcal{J}_{I}^{1}\right)$ exactly cancels the contribution from the gluon intrinsic-like part $\left(M \mathcal{J}_{I I I}^{1}\right)$.

The renormalized mass $m_{R}$ of the quark is given in terms of the bare mass up to order $\alpha_{s}$ in light-front Hamiltonian perturbation theory by [16]

$$
m_{R}=m\left(1+\frac{3 \alpha_{s}}{4 \pi} C_{f} \ln \frac{Q^{2}}{\mu^{2}}\right) .
$$

In the light-front formulation of QCD, there are two mass terms in the Hamiltonian, one is quadratic in $m$ which is present in the free part and does not break chiral symmetry, the other is linear in $m$ which we discuss here and which explicitly cause chiral symmetry breaking. An important feature of light-front QCD is that, these two mass scales are renormalized differently even in the perturbative region. The renormalization of $m^{2}$ is different from the result stated above.

Adding all the parts, for a dressed quark in perturbation theory up to $O\left(g^{2}\right)$, the expectation value of the transverse spin operator is given by

$$
\begin{aligned}
\left\langle P, S^{1}\left|M \mathcal{J}^{1}\right| P, S^{1}\right\rangle & =\left\langle P, S^{1}\left|M \mathcal{J}_{I}^{1}+M \mathcal{J}_{I I}^{1}+M \mathcal{J}_{I I I}^{1}\right| P, S^{1}\right\rangle \\
& =\frac{m_{R}}{2}
\end{aligned}
$$

It is important to mention that here we are calculating the expectation value of the operator $M \mathcal{J}^{i}$. In order to extract the eigenvalue of $\mathcal{J}^{i}$ one has to know the eigenvalue of $M$. Both $M \mathcal{J}^{i}$ and $M$ are dynamical operators. However, in this case, the mass $M$ in the LHS in the renormalized theory is nothing but the renormalized mass of the quark, which therefore gets canceled from the above equation, and we get

$$
\left\langle P, S^{1}\left|\mathcal{J}^{1}\right| P, S^{1}\right\rangle=\left\langle P, S^{1}\left|\mathcal{J}_{I}^{1}+\mathcal{J}_{I I}^{1}+\mathcal{J}_{I I I}^{1}\right| P, S^{1}\right\rangle=\frac{1}{2} .
$$

The identification of $\mathcal{J}$ with spin, therefore, requires knowledge of the mass eigenvalue, independently of the boost invariance properties of the light-front dynamics.

We can explicitly verify the relation between the integral of $g_{T}$ and the expectation value of the fermion intrinsic-like part of the transverse spin operator to order $\alpha_{s}$ in perturbative QCD. The transverse polarized structure function for a dressed quark is given [17] by

$$
\begin{aligned}
g_{T}\left(x, Q^{2}\right)= & \frac{e_{q}^{2}}{2} \frac{m}{S^{1}}\left\{\delta(1-x)+\frac{\alpha_{s}}{2 \pi} C_{f} \ln \frac{Q^{2}}{\mu^{2}}\right. \\
& \times\left[\frac{1+2 x-x^{2}}{1-x}-\delta(1-x) \int_{0}^{1} d x^{\prime} \frac{1+x^{\prime 2}}{1-x^{\prime}}\right. \\
& \left.\left.+\frac{1}{2} \delta(1-x)\right]\right\},
\end{aligned}
$$

so we get

$$
\int_{0}^{1} g_{T}(x) d x=\frac{e_{q}^{2}}{2 S^{1}}\left\langle P, S^{1}\left|M \mathcal{J}_{I I}^{1}\right| P, S^{1}\right\rangle
$$


which explicitly shows the connection between the integral of the transverse polarized structure function and the matrix element of the fermion intrinsic-like part of the transverse spin operator.

It is quite instructive to compare our calculation of the transverse spin of the dressed quark with the helicity of the dressed quark [7] in perturbative QCD. All the operators contributing to helicity are kinematical (interaction independent) and hence all of them give rise to only diagonal contributions. Further, in this calculation mass of the quark can be completely ignored since they give rise to only powersuppressed contribution. In the massless limit, helicity is conserved at the quark gluon vertex. This means that the quark in the quark-gluon state has the same helicity as the parent quark. Since the transverse gluon carry helicity \pm 1 , we get a non-vanishing contribution from the gluon intrinsic helicity operator. However, both the quark and the gluon in the quark-gluon state have non-vanishing orbital angular momentum due to transverse motion. Total helicity conservation implies that orbital contribution has to cancel gluon intrinsic helicity contribution. This is precisely what happens [7] and we find that the total quark plus gluon orbital part exactly canceled the intrinsic gluon contribution and the overall contribution to the helicity is $\pm \frac{1}{2}$, which entirely comes from the intrinsic part of the fermionic helicity operator.

In contrast, in the case of transverse spin operator, it has both interaction independent and interaction dependent parts. The latter gives rise to off-diagonal matrix elements and they play a very important role. Of special interest is the gluon intrinsic-like transverse spin operator. This operator gives vanishing matrix elements for a free gluon. However, since gluon in the quark-gluon state has intrinsic transverse momentum, both diagonal and off-diagonal terms give rise to non-vanishing contributions and we get a net non-vanishing matrix element for the gluon intrinsic-like transverse spin operator. However, we find that contribution from this matrix element is completely canceled by that from the matrix elements of orbital-like transverse spin operators. This is analogous to what happens in the helicity case.

In this section, the calculation of the matrix elements has been done in the frame with $P^{\perp}=0$. The complete calculation of the matrix element of the transverse spin operator in an arbitrary reference frame is given in Appendix D. It is clear from the expressions there that all the terms explicitly dependent on $P^{\perp}$ get canceled in the expectation value of $M \mathcal{J}^{1}$. The parts that remain after the cancellation of the $P^{\perp}$ dependent terms are those given above. In the above expressions, we have used the manifest Hermitian form of the operators. We again stress the fact that this manifest cancellation of contributions from center of mass motion is typical in light-front field theory because the transverse boost operators are kinematical. The situation in the equal time relativistic case is completely different and there one cannot separate out the center of mass motion from the internal motion in a straightforward way even in the free theory case [12] because of the complicated boost generators. Due to the manifest cancellation of the center of mass momenta, $\mathcal{J}^{i}$ can truly be identified as the transverse spin operator.

\section{SUMMARY AND CONCLUSIONS}

In this paper, we have analyzed the transverse spin operators in QCD. In equal time quantization, one encounters two major difficulties in the description of the spin of a composite system in an arbitrary reference frame. They are (1) the complicated interaction dependence arising from dynamical boost operators and (2) the difficulty in the separation of center of mass motion from the internal motion. Because of these severe difficulties, there have been hardly any attempt to study spin operators of a moving composite system in the conventional equal time formulation of quantum field theory.

In light-front theory, on the other hand, the longitudinal spin operator (light-front helicity) is interaction independent and the interaction dependence of transverse spin operators arises solely from that of transverse rotation operators. Moreover, in this case the separation of center of mass motion from internal motion is trivial since light-front transverse boosts are simple Galilean boosts.

It is extremely interesting to contrast the cases of longitudinal and transverse spin operators in light-front field theory. In the case of longitudinal spin operator (light-front helicity), in the gauge fixed theory, the operator is interaction independent and can be separated into orbital and spin parts for quarks and gluons. It is known for a long time that the transverse spin operators in light-front field theory cannot be separated into orbital and spin parts except in the trivial case of free field theory. In this work, we have shown that, in spite of the complexities, a physically interesting separation is indeed possible for the transverse spin operators which is quite different from the separation into orbital and spin parts in the rest frame familiar in the equal time picture. We have discussed the physical significance of this separation. Also transverse rotational symmetry is not manifest in light-front theory and a study of these operators is needed for questions regarding Lorentz invariance in the theory [14].

In analogy with the helicity sum rule, we have proposed a decomposition for the transverse spin. Elsewhere we have shown [9] the relationship between nucleon matrix elements of $\mathcal{J}_{I I}^{i}$ and $\mathcal{J}_{I I I}^{i}$ and the first moments of quark and gluon structure functions respectively, appearing in transverse polarized hard scattering. This is the first time that the integral of $g_{T}$ is related to a conserved quantity, namely the transverse spin operator. It is important to mention here that the proposed decomposition of the transverse spin operator will not be affected if one adds a total derivative term to the angular momentum density. Such a term can at most produce a surface term which we are neglecting since we have restricted ourselves to the topologically trivial sector of the theory. We have started with the angular momentum density defined in terms of the symmetric gauge invariant stressenergy tensor, which is obtained from the Noether's stressenergy tensor by a adding a total derivative term. Even though the angular momentum density differs from the Noether angular momentum density by a total derivative term, both give rise to the same generators. Another point worth mentioning is that we have worked in the gauge fixed theory. In the light-front gauge, $A^{+}=0$, the transverse spin operator can be separated into three parts, and $\mathcal{J}_{I I}^{i}$ is related to the 
first moment of $g_{T}$ measured in transverse polarized scattering, which is a gauge invariant object. This is similar to the helicity case, where only in the light-front gauge and using light-front quantization, the intrinsic fermionic helicity is related to the gauge invariant first moment of $g_{1}$ measured in longitudinally polarized scattering. The corresponding gluon intrinsic helicity cannot be measured directly in polarized deep inelastic lepton-nucleon scattering but in some other process like polarized hadron-hadron scattering. A similar situation holds in the case of transverse spin.

A very important issue related to the transverse spin operators is renormalization. Because of the interaction dependence, the operators acquire divergences in perturbation theory just like the Hamiltonian and therefore have to be renormalized. The renormalization of only the intrinsic-like fermion part of the transverse spin operator has been discussed in the literature so far. In this paper, we have carried out the renormalization of the full transverse spin operator for the first time up to $O\left(\alpha_{s}\right)$ in light-front Hamiltonian perturbation theory by evaluating the matrix elements for a dressed quark target. We have shown that the entire contribution to the matrix element comes from the fermion intrinsic-like part of the transverse spin operator and is equal to $\frac{1}{2}$. The contributions from $\mathcal{J}_{I}^{i}$ and $\mathcal{J}_{I I I}^{i}$ exactly get canceled. Also, the mass of the quark is very crucial in this case, since the helicity flip interactions which are proportional to the quark mass play a very important role. However, the terms proportional to $m^{2}$ do not flip the helicity and do not contribute. Since helicity flip is involved, we do not encounter any quadratic divergence unlike the case of renormalization of the light-front Hamiltonian. Further, we have compared and contrasted the calculations of transverse spin and helicity of a dressed quark in perturbation theory.

We have also verified the frame independence of our results. We have explicitly shown that, in an arbitrary reference frame, all the terms depending on the center of mass momenta manifestly get canceled in the matrix element. The cancellation is as simple as in non-relativistic theory since boost is kinematical on the light-front. For future studies, it is an interesting problem to evaluate non-perturbatively [18] the matrix element of the transverse spin operator in lightfront QCD. Also, in this work, we have used cutoff on the relative transverse momenta and the small $x$ divergence gets canceled in the one loop result. The renormalization of the transverse spin operators using similarity renormalization technique [10] is to be done in future.

\section{ACKNOWLEDGMENTS}

We thank the organizers of the Workshop on High Energy Physics Phenomenology-VI, Chennai, India, for warm hospitality where part of this work was done. We acknowledge helpful discussions with Professor P. P. Divakaran. R.R. gratefully acknowledges the financial assistance of the council of Scientific and Industrial Research (CSIR), India.

\section{APPENDIX A: POINCARE GENERATORS IN LIGHT-FRONT QCD}

In this appendix we derive the manifestly Hermitian kinematical Poincare generators (except $J^{3}$ ) and the Hamiltonian in light-front QCD starting from the gauge invariant symmetric energy momentum tensor $\Theta^{\mu \nu}$. To begin with, $\Theta^{\mu \nu}$ is interaction dependent. In the gauge fixed theory we find that the seven kinematical generators are manifestly independent of the interaction.

We shall work in the gauge $A^{+}=0$ and ignore all surface terms. Thus we are working in the completely gauge fixed sector of the theory [7]. The explicit form of the operator $J^{3}$ in this case is given in Ref. [7] which is manifestly free of interaction at the operator level. The rotation operators are given in Sec. II.

At $x^{+}=0$, the operators $K^{3}$ and $E^{i}$ depend only on the density $\Theta^{++}$. A straightforward calculation leads to

$$
\Theta^{++}=\psi^{+\dagger} i \stackrel{\leftrightarrow}{\partial^{+}} \psi^{+}+\partial^{+} A^{i} \partial^{+} A^{i}
$$

then, longitudinal momentum operator,

$$
\begin{aligned}
P^{+} & =\frac{1}{2} \int d x^{-} d^{2} x^{\perp} \Theta^{++} \\
& =\frac{1}{2} \int d x^{-} d^{2} x^{\perp}\left[\psi^{+\dagger} i \stackrel{\leftrightarrow}{\partial^{+}} \psi^{+}+\partial^{+} A^{j} \partial^{+} A^{j}\right],
\end{aligned}
$$

generator of longitudinal scaling,

$$
\begin{aligned}
K^{3} & =-\frac{1}{4} \int d x^{-} d^{2} x^{\perp} x^{-} \Theta^{++} \\
& =-\frac{1}{4} \int d x^{-} d^{2} x^{\perp} x^{-}\left[\psi^{+\dagger} i \stackrel{\leftrightarrow}{\partial} \psi^{+}+\partial^{+} A^{j} \partial^{+} A^{j}\right],
\end{aligned}
$$

transverse boost generators,

$$
\begin{aligned}
E^{i} & =-\frac{1}{2} \int d x^{-} d^{2} x^{\perp} x^{i} \Theta^{++} \\
& =-\frac{1}{2} \int d x^{-} d^{2} x^{\perp} x^{i}\left[\psi^{+\dagger} i \stackrel{\leftrightarrow}{\partial^{+}} \psi^{+}+\partial^{+} A^{j} \partial^{+} A^{j}\right] .
\end{aligned}
$$

and the transverse momentum operator

$$
P^{i}=\frac{1}{2} \int d x^{-} d^{2} x^{\perp} \Theta^{+i}
$$

which appears to have explicit interaction dependence. Using the constraint equations for $\psi^{-}$and $A^{-}$, we have

$$
\begin{aligned}
\Theta^{+i}= & \Theta_{F}^{+i}+\Theta_{G}^{+i}, \\
\Theta_{F}^{+i}= & 2 \psi^{+\dagger} i \partial^{i} \psi^{+}+2 g \psi^{+\dagger} A^{i} \psi^{+}, \\
\Theta_{G}^{+i}= & \partial^{+} A^{j} \partial^{i} A^{j}-\partial^{+} A^{j} \partial^{j} A^{i} \\
& +\partial^{+} A^{i} \partial^{j} A^{j}-2 g \psi^{+\dagger} A^{i} \psi^{+} .
\end{aligned}
$$

Thus 


$$
\begin{aligned}
P^{i}= & \frac{1}{2} \int d x^{-} d^{2} x^{\perp}\left[\psi^{+\dagger} i \stackrel{\leftrightarrow}{\partial^{i}} \psi^{+}+A^{j} \partial^{+} \partial^{j} A^{i}-A^{i} \partial^{+} \partial^{j} A^{j}\right. \\
& \left.-A^{j} \partial^{+} \partial^{i} A^{j}\right] .
\end{aligned}
$$

Thus we indeed verify that all the kinematical operators are explicitly independent of interactions.

Lastly, the Hamiltonian operator can be written in the manifestly Hermitian form as

$P^{-}=\frac{1}{2} \int d x^{-} d^{2} x^{\perp} \Theta^{+-}=\frac{1}{2} \int d x^{-} d^{2} x^{\perp}\left(\mathcal{H}_{0}+\mathcal{H}_{\text {int }}\right)$ where $\mathcal{H}_{0}$ is the free part given by

$$
\begin{aligned}
\mathcal{H}_{0}= & -A_{a}^{j}\left(\partial^{i}\right)^{2} A_{a}^{j}+\xi^{\dagger}\left[\frac{-\left(\partial^{\perp}\right)^{2}+m^{2}}{i \partial^{+}}\right] \xi \\
& -\left[\frac{-\left(\partial^{\perp}\right)^{2}+m^{2}}{i \partial^{+}} \xi^{\dagger}\right] \xi .
\end{aligned}
$$

The interaction terms are given by

$$
\mathcal{H}_{\text {int }}=\mathcal{H}_{\text {qqg }}+\mathcal{H}_{\text {ggg }}+\mathcal{H}_{\text {qqgg }}+\mathcal{H}_{q q q q}+\mathcal{H}_{\text {gggg }},
$$

where

$$
\begin{aligned}
\mathcal{H}_{q q g}= & -4 g \xi^{\dagger} \frac{1}{\partial^{+}}\left(\partial^{\perp} \cdot A^{\perp}\right) \xi+g \frac{\partial^{\perp}}{\partial^{+}}\left[\xi^{\dagger}\left(\tilde{\sigma}^{\perp} \cdot A^{\perp}\right)\right] \tilde{\sigma}^{\perp} \xi+g \xi^{\dagger}\left(\tilde{\sigma}^{\perp} \cdot A^{\perp}\right) \frac{1}{\partial^{+}}\left(\tilde{\sigma}^{\perp} \cdot \partial^{\perp}\right) \xi+g\left(\frac{\partial^{\perp}}{\partial^{+}} \xi^{\dagger}\right) \tilde{\sigma}^{\perp}\left(\tilde{\sigma}^{\perp} \cdot A^{\perp}\right) \xi+g \xi^{\dagger} \frac{1}{\partial^{+}} \\
& \times\left(\tilde{\sigma}^{\perp} \cdot \partial^{\perp}\right)\left(\tilde{\sigma}^{\perp} \cdot A^{\perp}\right) \xi-m g \frac{1}{\partial^{+}}\left[\xi^{\dagger}\left(\tilde{\sigma}^{\perp} \cdot A^{\perp}\right)\right] \xi+m g \xi^{\dagger}\left(\tilde{\sigma}^{\perp} \cdot A^{\perp}\right) \frac{1}{\partial^{+}} \xi+m g\left(\frac{1}{\partial^{+}} \xi^{\dagger}\right)\left(\tilde{\sigma}^{\perp} \cdot A^{\perp}\right) \xi-m g \xi^{\dagger} \frac{1}{\partial^{+}}\left[\left(\tilde{\sigma}^{\perp} \cdot A^{\perp}\right) \xi\right],
\end{aligned}
$$

$\mathcal{H}_{g g g}=2 g f^{a b c}\left[\partial^{i} A_{a}^{j} A_{b}^{i} A_{c}^{j}+\left(\partial^{i} A_{a}^{i}\right) \frac{1}{\partial^{+}}\left(A_{b}^{j} \partial^{+} A_{c}^{j}\right)\right]$,

$\mathcal{H}_{q q g g}=g^{2}\left[\xi^{\dagger}\left(\tilde{\sigma}^{\perp} \cdot A^{\perp}\right) \frac{1}{i \partial^{+}}\left(\tilde{\sigma}^{\perp} \cdot A^{\perp}\right) \xi-\frac{1}{i \partial^{+}}\left(\xi^{\dagger} \tilde{\sigma}^{\perp} \cdot A^{\perp}\right) \tilde{\sigma}^{\perp} \cdot A^{\perp} \xi+4 \frac{1}{\partial^{+}}\left(f^{a b c} A_{b}^{i} \partial^{+} A_{c}^{i}\right) \frac{1}{\partial^{+}}\left(\xi^{\dagger} T^{a} \xi\right)\right]$,

$\mathcal{H}_{q q q q}=4 g^{2} \frac{1}{\partial^{+}}\left(\xi^{\dagger} T^{a} \xi\right) \frac{1}{\partial^{+}}\left(\xi^{\dagger} T^{a} \xi\right)$

$\mathcal{H}_{g g g g}=\frac{g^{2}}{2} f^{a b c} f^{a d e}\left[A_{b}^{i} A_{c}^{j} A_{d}^{i} A_{e}^{j}+2 \frac{1}{\partial^{+}}\left(A_{b}^{i} \partial^{+} A_{c}^{i}\right) \frac{1}{\partial^{+}}\left(A_{d}^{j} \partial^{+} A_{e}^{j}\right)\right]$.

\section{APPENDIX B: TRANSVERSE SPIN FOR A SYSTEM OF TWO NON-INTERACTING FERMIONS}

In order to show the non-triviality of the transverse spin operators even in the free theory and the manifest cancellation of the center of mass motion in this case, here we evaluate the transverse spin for a composite system of two free fermions. The manifest cancellation of the center of mass motion for the interacting theory is much more complicated and is given in Appendix D.

Let the mass of each fermion be $m$ and momenta $\left(k_{i}^{+}, k_{i}^{\perp}\right), i=1,2$. We take the state to be $|P\rangle$ $=b^{\dagger}\left(k_{1}, s_{1}\right) b^{\dagger}\left(k_{2}, s_{2}\right)|0\rangle$, where $s_{1}$ and $s_{2}$ are the helicities.

$$
M \mathcal{J}^{1}|P\rangle=\left(\frac{1}{2} F^{2} P^{+}+K^{3} P^{2}-\frac{1}{2} E^{2} P^{-}-P^{1} \mathcal{J}^{3}\right)|P\rangle .
$$

We introduce Jacobi momenta, $\left(x_{i}, q^{\perp}\right)$ defined as

$$
k_{1}^{\perp}=q^{\perp}+x_{1} P^{\perp}, \quad k_{2}^{\perp}=-q^{\perp}+x_{2} P^{\perp}, \quad k_{i}^{+}=x_{i} P^{+}
$$

with $\sum x_{i}=1$.

Here $M$ is the mass of the composite system and $\left(P^{+}, P^{\perp}\right)$ are the momenta of the center of mass.

The partial derivatives with respect to the particle momenta can be expressed in terms of these variables as 


$$
\frac{\partial}{\partial k_{1}^{i}}=x_{2} \frac{\partial}{\partial q^{i}}+\frac{\partial}{\partial P^{i}}, \quad \frac{\partial}{\partial k_{2}^{i}}=-x_{1} \frac{\partial}{\partial q^{i}}+\frac{\partial}{\partial P^{i}},
$$

and

$$
\begin{aligned}
& \frac{\partial}{\partial k_{1}^{+}}=\frac{x_{2}}{P^{+}} \frac{\partial}{\partial x_{1}}+\frac{\partial}{\partial P^{+}}-x_{2} \frac{P^{\perp}}{P^{+}} \cdot \frac{\partial}{\partial q^{\perp}}, \\
& \frac{\partial}{\partial k_{2}^{+}}=\frac{x_{1}}{P^{+}} \frac{\partial}{\partial x_{2}}+\frac{\partial}{\partial P^{+}}+x_{1} \frac{P^{\perp}}{P^{+}} \cdot \frac{\partial}{\partial q^{\perp}} .
\end{aligned}
$$

Then we have

$$
K^{3} P^{2}|P\rangle=\left[-i P^{2} x_{1} x_{2}\left(\frac{\partial}{\partial x_{1}}+\frac{\partial}{\partial x_{2}}\right)-i P^{2} P^{+} \frac{\partial}{\partial P^{+}}\right]|P\rangle,
$$

$$
\left.+P^{1} \frac{s_{1}}{2}+P^{1} \frac{s_{2}}{2}\right]|P\rangle,
$$

$$
\begin{aligned}
\frac{1}{2} F^{2} P^{+}|P\rangle= & {\left[i q^{2}\left(x_{2} \frac{\partial}{\partial x_{1}}-x_{1} \frac{\partial}{\partial x_{2}}\right)+\frac{s_{1}}{2} \frac{q^{1}}{x_{1}}-\frac{s_{2}}{2} \frac{q^{1}}{x_{2}}+\frac{i}{2}\left(m^{2}+\left(q^{\perp}\right)^{2}\right)\left(\frac{x_{2}}{x_{1}}-\frac{x_{1}}{x_{2}}\right) \frac{\partial}{\partial q^{2}}+\frac{m}{2} \sum_{\lambda}\left(\frac{\sigma_{\lambda s_{1}}^{1}}{x_{1}}+\frac{\sigma_{\lambda s_{2}}^{1}}{x_{2}}\right)\right.} \\
& -i q^{2} P^{\perp} \cdot \frac{\partial}{\partial q^{\perp}}+i P^{2} x_{1} x_{2}\left(\frac{\partial}{\partial x_{1}}+\frac{\partial}{\partial x_{2}}\right)+i P^{+} P^{2} \frac{\partial}{\partial P^{+}}+\frac{i}{2}\left(m^{2}+\left(q^{\perp}\right)^{2}\right) \frac{1}{x_{1} x_{2}} \frac{\partial}{\partial P^{2}}+\frac{i}{2}\left(P^{\perp}\right)^{2} \frac{\partial}{\partial P^{2}} \\
& \left.+i\left(q^{\perp} \cdot P^{\perp}\right) \frac{\partial}{\partial q^{2}}+\frac{P^{1}}{2}\left(s_{1}+s_{2}\right)-i P^{2}\right]|P\rangle .
\end{aligned}
$$

Substituting

$$
M^{2}=\frac{\left(m^{2}+\left(q^{\perp}\right)^{2}\right)}{x_{1} x_{2}},
$$

we get

$$
\begin{aligned}
M \mathcal{J}^{1}|P\rangle= & {\left[i q^{2}\left(x_{2} \frac{\partial}{\partial x_{1}}-x_{1} \frac{\partial}{\partial x_{2}}\right)+\frac{i}{2}\left(m^{2}+\left(q^{\perp}\right)^{2}\right)\right.} \\
& \times\left(\frac{x_{2}}{x_{1}}-\frac{x_{1}}{x_{2}}\right) \frac{\partial}{\partial q^{2}} \\
& \left.+\frac{q^{1}}{2}\left(\frac{s_{1}}{x_{1}}-\frac{s_{2}}{x_{2}}\right)+\frac{m}{2} \sum_{\lambda}\left(\frac{\sigma_{\lambda s_{1}}^{1}}{x_{1}}+\frac{\sigma_{\lambda s_{2}}^{1}}{x_{2}}\right)\right]|P\rangle .
\end{aligned}
$$

Explicitly we see that $M \mathcal{J}^{1}$ does not depend on the center of mass momenta.

\section{APPENDIX C: TRANSVERSE ROTATION OPERATOR IN QCD}

In this section we explicitly derive the expressions for interaction dependent transverse rotation operators in lightfront QCD starting from the manifestly gauge invariant energy momentum tensor.

We set the gauge $A^{+}=0$ and eliminate the dependent variables $\psi^{-}$and $A^{-}$using the equations of constraint. In this paper we restrict to the topologically trivial sector of the theory and set the boundary condition $A^{i}\left(x^{-}, x^{i}\right) \rightarrow 0$ as $x^{-, i} \rightarrow \infty$. This completely fixes the gauge and sets all surface terms to zero.

The transverse rotation operator

$$
F^{i}=\frac{1}{2} \int d x^{-} d^{2} x^{\perp}\left[x^{-} \Theta^{+i}-x^{i} \Theta^{+-}\right] .
$$

The symmetric gauge invariant energy momentum tensor

$$
\begin{aligned}
\Theta^{\mu \nu}= & \frac{1}{2} \bar{\psi}\left[\gamma^{\mu} i D^{\nu}+\gamma^{\nu} i D^{\mu}\right] \psi-F^{\mu \lambda a} F_{\lambda}^{\nu a}-g^{\mu \nu} \\
& \times\left[-\frac{1}{4}\left(F_{\lambda \sigma a}\right)^{2}+\bar{\psi}\left(\gamma^{\lambda} i D_{\lambda}-m\right) \psi\right],
\end{aligned}
$$

where

$$
\begin{aligned}
i D^{\mu} & =\frac{1}{2} i \stackrel{\leftrightarrow}{\partial^{\mu}}+g A^{\mu}, \\
F^{\mu \lambda a} & =\partial^{\mu} A^{\lambda a}-\partial^{\lambda} A^{\mu a}+g f^{a b c} A^{\mu b} A^{\lambda c}, \\
F_{\lambda}^{\nu a} & =\partial^{\nu} A_{\lambda}^{a}-\partial_{\lambda} A^{\nu a}+g f^{a b c} A^{\nu b} A_{\lambda}^{c} .
\end{aligned}
$$

First consider the fermionic part of $\Theta^{\mu \nu}$ :

$$
\Theta_{F}^{\mu \nu}=\frac{1}{2} \bar{\psi}\left[\gamma^{\mu} i D^{\nu}+\gamma^{\nu} i D^{\mu}\right] \psi-g^{\mu \nu} \bar{\psi}\left(\gamma^{\lambda} i D_{\lambda}-m\right) \psi .
$$


The coefficient of $g^{\mu \nu}$ vanishes because of the equation of motion.

Explicitly, the contribution to $F^{2}$ from the fermionic part of $\Theta^{\mu \nu}$ is given by

$$
\begin{aligned}
F_{F}^{2}= & \frac{1}{2} \int d x^{-} d^{2} x^{\perp}\left[x^{-} \frac{1}{2} \bar{\psi}\left(\gamma^{+} i D^{2}+\gamma^{2} i D^{+}\right) \psi\right. \\
& \left.-x^{2} \frac{1}{2} \bar{\psi}\left(\gamma^{+} i D^{-}+\gamma^{-} i D^{+}\right) \psi\right] \\
= & F_{F(I)}^{2}+F_{F(I I)}^{2},
\end{aligned}
$$

where

$$
\begin{aligned}
F_{F(I)}^{2}= & \frac{1}{2} \int d x^{-} d^{2} x^{\perp} x^{-}\left[\psi^{+\dagger} \frac{1}{2} i \stackrel{\leftrightarrow}{\partial}^{2} \psi^{+}+\psi^{+\dagger} g A^{2} \psi^{+}\right. \\
& \left.+\frac{1}{4} \bar{\psi} \gamma^{i} i \stackrel{\leftrightarrow}{\partial}^{+} \psi\right],
\end{aligned}
$$

$$
\begin{aligned}
F_{F(I I)}^{2}= & -\frac{1}{2} \int d x^{-} d^{2} x^{\perp} x^{2}\left[\psi^{+\dagger}\left(\frac{1}{2} i \overleftrightarrow{\partial}^{-}+g A^{-}\right) \psi^{+}\right. \\
& \left.+\frac{1}{4} \psi^{-\dagger} \gamma^{i} i \stackrel{\leftrightarrow}{\partial}^{+} \psi^{-}\right] .
\end{aligned}
$$

We have the equation of constraint

$$
i \partial^{+} \psi^{-}=\left[\alpha^{\perp} \cdot\left(i \partial^{\perp}+g A^{\perp}\right)+\gamma^{0} m\right] \psi^{+},
$$

and the equation of motion

$$
\begin{aligned}
i \partial^{-} \psi^{+}= & -g A^{-} \psi^{+}+\left[\alpha^{\perp} \cdot\left(i \partial^{\perp}+g A^{\perp}\right)+\gamma^{0} m\right] \frac{1}{i \partial^{+}} \\
& \times\left[\alpha^{\perp} \cdot\left(i \partial^{\perp}+g A^{\perp}\right)+\gamma^{0} m\right] \psi^{+} .
\end{aligned}
$$

Using the Eqs. (C8) and (C9) we arrive at free ( $g$ independent) and interaction ( $g$ dependent) parts of $F_{F}^{2}$. The free part of $F_{F}^{2}$ is given by

$$
\begin{aligned}
F_{F(\text { free })}^{2}= & \frac{1}{2} \int d x^{-} d^{2} x^{\perp}\left\{x^{-}\left[\xi^{\dagger}\left[i \partial^{2} \xi\right]-\left[i \partial^{2} \xi^{\dagger}\right] \xi\right]-x^{2}\left[\xi^{\dagger}\left[\frac{-\left(\partial^{\perp}\right)^{2}+m^{2}}{i \partial^{+}} \xi\right]-\left[\frac{-\left(\partial^{\perp}\right)^{2}+m^{2}}{i \partial^{+}} \xi^{\dagger}\right] \xi\right]\right. \\
& \left.+\left[\xi^{\dagger}\left[\sigma^{3} \partial^{1}+i \partial^{2}\right] \frac{1}{\partial^{+}} \xi+\left[\frac{1}{\partial^{+}}\left(\partial^{1} \xi^{\dagger} \sigma^{3}-i \partial^{2} \xi^{\dagger}\right)\right] \xi\right]+m\left[\xi^{\dagger}\left[\frac{\sigma^{1}}{i \partial^{+}} \xi\right]-\left[\frac{1}{i \partial^{+}} \xi^{\dagger} \sigma^{1}\right] \xi\right]\right\} .
\end{aligned}
$$

We have introduced the two-component field $\xi$,

$$
\psi^{+}=\left[\begin{array}{c}
\xi \\
0
\end{array}\right]
$$

The interaction dependent part of $F_{F(I)}^{2}$ is

$$
\begin{aligned}
F_{F(I) i n t}^{2}= & g \int d x^{-} d^{2} x^{\perp} x^{-} \xi^{\dagger} A^{2} \xi+\frac{1}{4} g \int d x^{-} d^{2} x^{\perp} \\
& \times\left[\xi^{\dagger} \frac{1}{\partial^{+}}\left[\left(-i \sigma^{3} A^{1}+A^{2}\right) \xi\right]+\frac{1}{\partial^{+}}\left[\xi^{\dagger}\left(i \sigma^{3} A^{1}+A^{2}\right)\right] \xi\right] .
\end{aligned}
$$

The interaction dependent part of $F_{F(I I)}^{2}$ is

$$
\begin{aligned}
F_{F(I I) i n t}^{2}= & \frac{1}{4} g \int d x^{-} d^{2} x^{\perp}\left[\xi^{\dagger} \frac{1}{\partial^{+}}\left[\left(-i \sigma^{3} A^{1}+A^{2}\right) \xi\right]+\frac{1}{\partial^{+}}\left[\xi^{\dagger}\left(i \sigma^{3} A^{1}+A^{2}\right)\right] \xi\right] \\
& -\frac{1}{2} g \int d x^{-} d^{2} x^{\perp} x^{2}\left[\frac{\partial^{\perp}}{\partial^{+}}\left[\xi^{\dagger}\left(\tilde{\sigma}^{\perp} \cdot A^{\perp}\right)\right] \tilde{\sigma}^{\perp} \xi+\xi^{\dagger}\left(\tilde{\sigma}^{\perp} \cdot A^{\perp}\right) \frac{1}{\partial^{+}}\left(\tilde{\sigma}^{\perp} \cdot \partial^{\perp}\right) \xi\right. \\
& +\left(\frac{\partial^{\perp}}{\partial^{+}} \xi^{\dagger}\right) \tilde{\sigma}^{\perp}\left(\tilde{\sigma}^{\perp} \cdot A^{\perp}\right) \xi+\xi^{\dagger} \frac{1}{\partial^{+}}\left(\tilde{\sigma}^{\perp} \cdot \partial^{\perp}\right)\left(\tilde{\sigma}^{\perp} \cdot A^{\perp}\right) \xi-m \frac{1}{\partial^{+}}\left[\xi^{\dagger}\left(\tilde{\sigma}^{\perp} \cdot A^{\perp}\right)\right] \xi+m \xi^{\dagger}\left(\tilde{\sigma}^{\perp} \cdot A^{\perp}\right) \frac{1}{\partial^{+}} \xi \\
& \left.+m\left(\frac{1}{\partial^{+}} \xi^{\dagger}\right)\left(\tilde{\sigma}^{\perp} \cdot A^{\perp}\right) \xi-m \xi^{\dagger} \frac{1}{\partial^{+}}\left[\left(\tilde{\sigma}^{\perp} \cdot A^{\perp}\right) \xi\right]\right]-\frac{1}{2} g^{2} \int d x^{-} d^{2} x^{\perp} x^{2}\left[\xi^{\dagger} \tilde{\sigma}^{\perp} \cdot A^{\perp} \frac{1}{i \partial^{+}} \tilde{\sigma}^{\perp} \cdot\left(A^{\perp} \xi\right)\right. \\
& \left.-\frac{1}{i \partial^{+}}\left(\xi^{\dagger} \tilde{\sigma}^{\perp} \cdot A^{\perp}\right) \tilde{\sigma}^{\perp} \cdot A^{\perp} \xi\right] .
\end{aligned}
$$


We have introduced $\tilde{\sigma}^{1}=\sigma^{2}$ and $\tilde{\sigma}^{2}=-\sigma^{1}$.

Next consider the gluonic part of the operator $F^{2}$ :

$$
F_{g}^{2}=\frac{1}{2} \int d x^{-} d^{2} x^{\perp}\left[x^{-} \Theta_{g}^{+2}-x^{2} \Theta_{g}^{+-}\right]
$$

where

$$
\begin{aligned}
& \Theta_{g}^{+2}=-F^{+\lambda a} F_{\lambda}^{2 a}, \\
& \Theta_{g}^{+-}=-F^{+\lambda a} F_{\lambda}^{-a}+\frac{1}{4} g^{+-}\left(F_{\lambda \sigma a}\right)^{2} .
\end{aligned}
$$

Using the constraint equation

$$
\frac{1}{2} \partial^{+} A^{-a}=\partial^{i} A^{i a}+g f^{a b c} \frac{1}{\partial^{+}}\left(A^{i b} \partial^{+} A^{i c}\right)+2 g \frac{1}{\partial^{+}}\left(\xi^{\dagger} T^{a} \xi\right),
$$

we arrive at

$$
F_{g}^{2}=F_{g(\text { free })}^{2}+F_{g(\text { int })}^{2}
$$

where

$$
\begin{aligned}
F_{g(\text { free })}^{2}= & \frac{1}{2} \int d x^{-} d^{2} x^{\perp}\left\{x ^ { - } \left(A^{j a} \partial^{+} \partial^{j} A^{2 a}-A^{2 a} \partial^{+} \partial^{j} A^{j a}\right.\right. \\
& \left.\left.+A^{j a} \partial^{+} \partial^{2} A^{j a}\right)-x^{2}\left(A^{k a}\left(\partial^{j}\right)^{2} A^{k a}\right)\right\} \\
& -2 \int d x^{-} d^{2} x^{\perp} A^{2 a} \partial^{1} A^{1 a}
\end{aligned}
$$

$$
\begin{aligned}
F_{g(\text { int })}^{2}= & \frac{1}{2} \int d x^{-} d^{2} x^{\perp} x^{-}\left\{g f^{a b c} \partial^{+} A^{i a} A^{2 b} A^{i c}+g\left(f^{a b c} \frac{1}{\partial^{+}}\left(A^{i b} \partial^{+} A^{i c}\right)+2 \frac{1}{\partial^{+}}\left(\xi^{\dagger} T^{a} \xi\right)\right) \partial^{+} A^{2 a}\right\} \\
& -\frac{1}{2} \int d x^{-} d^{2} x^{\perp} x^{2}\left\{2 g f^{a b c} \partial^{i} A^{j a} A^{i b} A^{j c}+\frac{g^{2}}{2} f^{a b c} f^{a d e} A^{i b} A^{j c} A^{i d} A^{j e}+2 g \partial^{i} A^{i a} \frac{1}{\partial^{+}}\left(f^{a b c} A^{j b} \partial^{+} A^{j c}+2 \xi^{\dagger} T^{a} \xi\right)\right. \\
& \left.+g^{2}\left(f^{a b c} \frac{1}{\partial^{+}}\left(A^{i b} \partial^{+} A^{i c}\right)+2 \frac{1}{\partial^{+}} \xi^{\dagger} T^{a} \xi\right)\left(f^{a d e} \frac{1}{\partial^{+}}\left(A^{j d} \partial^{+} A^{j e}\right)+2 \frac{1}{\partial^{+}} \xi^{\dagger} T^{a} \xi\right)\right\} .
\end{aligned}
$$

So the full transverse rotation operator in QCD can be written as

$$
F^{2}=F_{I}^{2}+F_{I I}^{2}+F_{I I I}^{2}
$$

where the explicit forms of $F_{I}^{2}, F_{I I}^{2}$ and $F_{I I I}^{2}$ have been given in Sec. II.

\section{APPENDIX D: TRANSVERSE SPIN OF A DRESSED QUARK IN AN ARBITRARY REFERENCE FRAME}

We introduce a wave packet state

$$
\left|\psi_{\sigma}\right\rangle=\frac{1}{2} \int d P^{+} d^{2} P^{\perp} f(P)|P, \sigma\rangle
$$

which is normalized as

$$
\left\langle\psi_{\sigma} \mid \psi_{\sigma^{\prime}}\right\rangle=\delta_{\sigma \sigma^{\prime}}
$$

Here $f(P)$ is a function of $P$, the exact form of which is not important. Using Eq. (4.2) we get

$$
\frac{1}{2} \int d P^{+} d^{2} P^{\perp} f^{*}(P) f(P)(2 \pi)^{3} P^{+}=1 .
$$

The expectation values of the various operators involved in the definition of $M \mathcal{J}^{i}$ are given below. It is to be noted that we have done the calculation in an arbitrary reference frame, in order to show that the dependence on the total center of mass momenta $\left(P^{+}, P^{\perp}\right)$ actually gets canceled in the expectation value of $M \mathcal{J}^{i}$.

The matrix elements presented below have been evaluated between wave packet states of different helicities, namely $\sigma$ and $\sigma^{\prime}$. Since the transversely polarized state can be expressed in terms of the longitudinally polarized (helicity) states by Eq. (4.3), the matrix elements of these operators between transversely polarized states can be easily obtained from these expressions. We introduce

$$
\psi_{1}^{\sigma}=f(P) \Phi_{1}^{\sigma}, \quad \psi_{\sigma_{1} \lambda}^{\sigma}=f(P) \Phi_{\sigma_{1} \lambda}^{\sigma}
$$

The matrix elements are given by 


$$
\begin{aligned}
\left\langle\psi_{\sigma}\left|K^{3} P^{2}\right| \psi_{\sigma^{\prime}}\right\rangle= & \frac{1}{2} \int d P^{+} d^{2} P^{\perp}(2 \pi)^{3} P^{+}\left(\frac{i}{2} \psi_{1}^{* \sigma} \frac{\partial \psi_{1}^{\sigma^{\prime}}}{\partial P^{+}} P^{+} P^{2}+\frac{i}{2} \sum_{s p i n} \int d x d^{2} q^{\perp} P^{2} \psi_{\sigma_{1} \lambda}^{\sigma *} \frac{\partial \psi_{\sigma_{1}^{\prime} \lambda^{\prime}}^{\sigma^{\prime}}}{\partial P^{+}} P^{+}+\text {H.c. }\right), \\
\left\langle\psi_{\sigma}\left|\frac{1}{2} E^{2} P_{\text {free }}^{-}\right| \psi_{\sigma^{\prime}}\right\rangle= & \frac{1}{2} \int d P^{+} d^{2} P^{\perp}(2 \pi)^{3} P^{+}\left(-\frac{i}{4} \psi_{1}^{* \sigma} \frac{\partial \psi_{1}^{\sigma^{\prime}}}{\partial P^{2}} P^{+} \frac{\left(P^{\perp}\right)^{2}+m^{2}}{P^{+}}\right. \\
& \left.-\frac{i}{4} \sum_{\text {spin }} \int d x d^{2} q^{\perp} \psi_{\sigma_{1} \lambda}^{* \sigma} \frac{\partial \psi_{\sigma_{1}^{\prime} \lambda^{\prime}}^{\sigma^{\prime}}}{\partial P^{2}}\left[\frac{m^{2}+\left(q^{\perp}+x P^{\perp}\right)^{2}}{x}+\frac{\left(-q^{\perp}+(1-x) P^{\perp}\right)^{2}}{1-x}\right]+\text { H.c. }\right), \\
\left\langle\psi_{\sigma}\left|\frac{1}{2} E^{2} P_{\text {int }}^{-}\right| \psi_{\sigma^{\prime}}\right\rangle= & -\frac{g}{\sqrt{2(2 \pi)^{3}}} \frac{1}{2} \int d P^{+} d^{2} P^{\perp}(2 \pi)^{3} P^{+} \sum_{\text {spin }} \int d x d^{2} q^{\perp} \frac{1}{\sqrt{1-x}}\left(i \psi _ { 1 } ^ { \sigma * } \frac { \partial \psi _ { \sigma _ { 1 } \lambda } ^ { \sigma ^ { \prime } } } { \partial P ^ { 2 } } \chi _ { \sigma } ^ { \dagger } \left[-\frac{\left(q^{\perp} \cdot \epsilon^{\perp}\right)}{1-x}\right.\right. \\
& \left.\left.-\frac{1\left(\tilde{\sigma}^{\perp} \cdot \epsilon^{\perp}\right)\left(\tilde{\sigma}^{\perp} \cdot q^{\perp}\right)}{2}-\frac{1}{2} i m \frac{(1-x)}{x}\left(\tilde{\sigma}^{\perp} \cdot \epsilon^{\perp}\right)\right] \chi_{\sigma_{1}}+\text { H.c. }\right) .
\end{aligned}
$$

Here H.c. is the Hermitian conjugate, $\Sigma_{\text {spin }}$ is summation over $\sigma_{1}, \sigma_{1}^{\prime}, \lambda, \lambda^{\prime} . P_{\text {free }}^{-}$is the free part and $P_{\text {int }}^{-}$is the interaction part of the light-front QCD Hamiltonian density.

$$
\begin{aligned}
\left\langle\psi_{\sigma}\left|P^{1} \mathcal{J}^{3}\right| \psi \sigma^{\prime}\right\rangle= & \frac{1}{2} \int d P^{+} d^{2} P^{\perp}(2 \pi)^{3} P^{+}\left[\sum_{s p i n} \int d x d^{2} q^{\perp} P^{1}\left(\frac{i}{2} \psi_{\sigma_{1} \lambda}^{* \sigma}\left(q^{2} \frac{\partial}{\partial q^{1}}-q^{1} \frac{\partial}{\partial q^{2}}\right) \psi_{\sigma_{1}^{\prime} \lambda^{\prime}}^{\sigma^{\prime}}+\text { H.c. }\right)\right. \\
& \left.+\frac{1}{2} \int d x d^{2} q^{\perp} P^{1} \sum_{\lambda, \sigma_{2}, \sigma_{2}^{\prime}} \lambda \psi_{\lambda \sigma_{2}}^{* \sigma} \psi_{\lambda \sigma_{2}^{\prime}}^{\sigma^{\prime}}+\int d x d^{2} q^{\perp} P^{1} \sum_{\lambda, \sigma_{1}, \sigma_{1}^{\prime}} \lambda \psi_{\sigma_{1} \lambda}^{* \sigma} \psi_{\sigma_{1}^{\prime} \lambda}^{\sigma^{\prime}}\right] .
\end{aligned}
$$

The first term in the above expression is the quark-gluon orbital part, the second and the third terms are the intrinsic helicities of the quark and gluon, respectively. Finally, the operator $\frac{1}{2} F^{2} P^{+}$can be separated into three parts,

$$
\frac{1}{2} F^{2} P^{+}=\frac{1}{2} F_{I}^{2} P^{+}+\frac{1}{2} F_{I I}^{2} P^{+}+\frac{1}{2} F_{I I I}^{2} P^{+}
$$

where $F_{I}^{2}, F_{I I}^{2}$ and $F_{I I I}^{2}$ have been defined earlier. The matrix elements of the different parts of these operators for a dressed quark state in an arbitrary reference frame are given below. A part of this calculation has been given in some detail in Appendix E. We have

$$
\frac{1}{2} F_{I}^{2} P^{+}=\frac{1}{2} F_{I}^{2}(1) P^{+}-\frac{1}{2} F_{I}^{2}(2) P^{+}-\frac{1}{2} F_{I}^{2}(3) P^{+}
$$

The matrix elements of these three parts are

$$
\begin{aligned}
\left\langle\psi_{\sigma}\left|\frac{1}{2} F_{I}^{2}(1) P^{+}\right| \psi_{\sigma^{\prime}}\right\rangle= & \left\langle\psi_{\sigma}\left|\frac{1}{2} \int d x d^{2} q^{\perp} x^{-} P_{0}^{2} \frac{1}{2} P^{+}\right| \psi_{\sigma^{\prime}}\right\rangle \\
= & \frac{1}{2} \int d P^{+} d^{2} P^{\perp}(2 \pi)^{3} P^{+}\left[-\frac{i}{2} \psi_{1}^{* \sigma} \frac{\partial \psi_{1}^{\sigma^{\prime}}}{\partial P^{+}} P^{+} P^{2}+\frac{i}{2} \sum_{s p i n} \int d x d^{2} q^{\perp} q^{2} \psi_{\sigma_{1} \lambda}^{* \sigma} P^{\perp} \frac{\partial \psi_{\sigma_{1}^{\prime} \lambda^{\prime}}^{\sigma^{\prime}}}{\partial q^{\perp}}\right. \\
& \left.-\frac{i}{2} \sum_{\text {spin }} \int d x d^{2} q^{\perp} \psi_{\sigma_{1} \lambda}^{* \sigma} P^{2} \frac{\partial \psi_{\sigma_{1}^{\prime} \lambda^{\prime}}^{\sigma^{\prime}}}{\partial P^{+}} P^{+}-\frac{i}{2} \sum_{\text {spin }} \int d x d^{2} q^{\perp} q^{2} \psi_{\sigma_{1} \lambda}^{* \sigma} \frac{\partial \psi_{\sigma_{1}^{\prime} \lambda^{\prime}}^{* \sigma^{\prime}}}{\partial x}+\text { H.c. }\right],
\end{aligned}
$$




$$
\begin{aligned}
\left\langle\psi_{\sigma}\left|\frac{1}{2} F_{I}^{2}(2) P^{+}\right| \psi_{\sigma^{\prime}}\right\rangle= & \left\langle\psi_{\sigma}\left|\frac{1}{2} \int d x d^{2} q^{\perp} x^{2} P_{0}^{-} \frac{1}{2} P^{+}\right| \psi_{\sigma^{\prime}}\right\rangle \\
= & \frac{1}{2} \int d P^{+} d^{2} P^{\perp}(2 \pi)^{3} P^{+}\left[\frac{i}{4} \psi_{1}^{* \sigma} \frac{\partial \psi_{1}^{\sigma^{\prime}}}{\partial P^{2}} \frac{\left(P^{\perp}\right)^{2}+m^{2}}{P^{+}} P^{+}+\frac{i}{4} \sum_{s p i n} \int d x d^{2} q^{\perp} \psi_{\sigma_{1} \lambda}^{* \sigma} \frac{\partial \psi_{\sigma_{1}^{\prime} \lambda^{\prime}}^{\sigma^{\prime}}}{\partial P^{2}}\right. \\
& \times\left[\frac{m^{2}+\left(q^{\perp}+x P^{\perp}\right)^{2}}{x}+\frac{\left(-q^{\perp}+(1-x) P^{\perp}\right)^{2}}{1-x}\right]+\frac{i}{2} \sum_{s p i n} \int d x d^{2} q^{\perp} \psi_{\sigma_{1} \lambda}^{* \sigma} \frac{\partial \psi_{\sigma_{1}^{\prime} \lambda^{\prime}}^{\sigma^{\prime}}}{\partial q^{2}}\left(q^{\perp} \cdot P^{\perp}\right) \\
& \left.+\frac{i}{4} \sum_{\text {spin }} \int d x d^{2} q^{\perp} \psi_{\sigma_{1} \lambda}^{* \sigma} \frac{\partial \psi_{\sigma_{1}^{\prime} \lambda^{\prime}}^{\sigma^{\prime}}}{\partial q^{2}}\left(q^{\perp}\right)^{2}\left(\frac{1-x}{x}-\frac{x}{1-x}\right)+\frac{i}{4} \sum_{\text {spin }} \int d x d^{2} q^{\perp} m^{2} \frac{1-x}{x} \psi_{\sigma_{1} \lambda}^{* \sigma} \frac{\partial \psi_{\sigma_{1}^{\prime} \lambda^{\prime}}^{\sigma^{\prime}}}{\partial q^{2}}+\text { H.c. }\right]
\end{aligned}
$$

In the above two equations, both the single particle and two particle diagonal matrix elements contribute.

$$
\begin{aligned}
\left\langle\psi_{\sigma}\left|\frac{1}{2} F_{I}^{2}(3) P^{+}\right| \psi_{\sigma^{\prime}}\right\rangle= & \left\langle\psi_{\sigma}\left|\frac{1}{2} \int d x d^{2} q^{\perp} x^{2} P_{\text {int }}^{-} \frac{1}{2} P^{+}\right| \psi_{\sigma^{\prime}}\right\rangle \\
= & \frac{g}{\sqrt{2(2 \pi)^{3}}} \sum_{\text {spin }} \frac{1}{2} \int d P^{+} d^{2} P^{\perp}(2 \pi)^{3} P^{+} \int d x d^{2} q^{\perp} \frac{1}{\sqrt{1-x}}\left(i \psi_{1}^{\sigma *} \frac{\partial \psi_{\sigma_{1} \lambda}^{\sigma^{\prime}}}{\partial P^{2}} \chi_{\sigma}^{\dagger}\right. \\
& \times\left[-\frac{\left(q^{\perp} \cdot \epsilon^{\perp}\right)}{1-x}-\frac{1}{2} \frac{\left(\tilde{\sigma}^{\perp} \cdot \epsilon^{\perp}\right)\left(\tilde{\sigma}^{\perp} \cdot q^{\perp}\right)}{x}-\frac{1}{2} i m \frac{(1-x)}{x}\left(\tilde{\sigma}^{\perp} \cdot \epsilon^{\perp}\right)\right] \chi_{\sigma_{1}} \\
& \left.-\frac{i}{4} \psi^{* \sigma} \chi_{\sigma}^{\dagger}\left[\tilde{\sigma}^{2}\left(\tilde{\sigma}^{\perp} \cdot \epsilon^{\perp}\right)+\frac{\left(\tilde{\sigma}^{\perp} \cdot \epsilon^{\perp}\right) \tilde{\sigma}^{2}}{x}\right] \chi_{\sigma_{1}} \psi_{\sigma_{1} \lambda}^{\sigma^{\prime}}+\text { H.c. }\right) .
\end{aligned}
$$

Only the off-diagonal matrix elements contribute in the above equation. The matrix elements of the three different parts of $\frac{1}{2} F_{I I}^{2} P^{+}$are given by,

$$
\begin{aligned}
& \left\langle\psi_{\sigma}\left|\frac{1}{2} F_{m I I}^{2} P^{+}\right| \psi_{\sigma^{\prime}}\right\rangle \\
& =\frac{1}{2} \int d P^{+} d^{2} P^{\perp}(2 \pi)^{3} P^{+}\left[\frac{m}{2} \psi_{1}^{* \sigma} \psi_{1}^{\sigma^{\prime}}+\frac{m}{2} \sum_{\text {spin }} \int d x d^{2} q^{\perp} \psi_{\sigma_{1} \lambda}^{* \sigma} \chi_{\sigma_{1}}^{\dagger} \sigma^{1} \chi_{\sigma_{1}^{\prime}} \psi_{\sigma_{1}^{\prime} \lambda^{\prime}}^{\sigma^{\prime}} \frac{1}{x}\right] \\
& \left\langle\psi_{\sigma}\left|\frac{1}{2} F_{q^{\perp} I I}^{2} P^{+}\right| \psi_{\sigma^{\prime}}\right\rangle \\
& =\frac{1}{2} \int d P^{+} d^{2} P^{\perp}(2 \pi)^{3} P^{+}\left[\frac{1}{2} \sum_{\text {spin }} \int d x d^{2} q^{\perp} \psi_{\sigma_{1} \lambda}^{* \sigma} \chi_{\sigma_{1}}^{\dagger} \sigma^{3} q^{1} \chi_{\sigma_{1}^{\prime}} \psi_{\sigma_{1}^{\prime} \lambda^{\prime}}^{\sigma^{\prime}} \frac{1}{x}\right. \\
& \left.\quad+\frac{1}{2} \int d x d^{2} q^{\perp} \sum_{\lambda, \sigma_{2}, \sigma_{2}^{\prime}} \lambda P^{1} \psi_{\lambda \sigma_{2}}^{* \sigma} \psi_{\lambda \sigma_{2}^{\prime}}^{\sigma^{\prime}}\right]
\end{aligned}
$$




$$
\begin{aligned}
\left\langle\psi_{\sigma}\right| & \frac{1}{2} F_{g I I}^{2} P^{+}\left|\psi_{\sigma^{\prime}}\right\rangle \\
= & \frac{1}{4} \frac{g}{\sqrt{2(2 \pi)^{3}}} \sum_{\operatorname{spin}} \frac{1}{2} \int d P^{+} d^{2} P^{\perp}(2 \pi)^{3} P^{+} \int d x d^{2} q^{\perp} \frac{1}{\sqrt{1-x}}\left(i \psi _ { 1 } ^ { * \sigma } \left[\chi_{\sigma}^{\dagger}\left(-i \sigma^{3} \epsilon_{\lambda}^{1}+\epsilon_{\lambda}^{2}\right) \chi_{\sigma_{1}}\right.\right. \\
& \left.\left.-\frac{1}{x} \chi_{\sigma}^{\dagger}\left(i \sigma^{3} \epsilon_{\lambda}^{1}+\epsilon_{\lambda}^{2}\right) \chi_{\sigma_{1}}\right] \psi_{\sigma_{1} \lambda}^{\sigma^{\prime}}+\text { H.c. }\right)
\end{aligned}
$$

In Eqs. (D14) and (D15), contributions come from only diagonal matrix elements whereas Eq. (D16) contain only off-diagonal matrix elements. The matrix element of $\frac{1}{2} F_{I I I}^{2} P^{+}$is given by

$$
\begin{aligned}
\left\langle\psi_{\sigma}\left|\frac{1}{2} F_{I I I}^{2} P^{+}\right| \psi_{\sigma^{\prime}}\right\rangle= & -\frac{g}{\sqrt{2(2 \pi)^{3}}} \frac{1}{2} \int d P^{+} d^{2} P^{\perp}(2 \pi)^{3} P^{+}\left[\sum_{s p i n} \int d x d^{2} q^{\perp} \frac{1}{\sqrt{1-x}}\left(\psi_{1}^{* \sigma} \epsilon_{\lambda}^{2} \psi_{\sigma_{1} \lambda}^{\sigma^{\prime}} \frac{1}{i(1-x)}+\mathrm{H} . c .\right)\right. \\
& \left.-\int d x d^{2} q^{\perp} \frac{q^{1}}{(1-x)} \sum_{\lambda, \sigma_{1}, \sigma_{1}^{\prime}} \lambda \psi_{\sigma_{1} \lambda}^{* \sigma} \psi_{\sigma_{1}^{\prime} \lambda}^{\sigma^{\prime}}+\int d x d^{2} q^{\perp} P^{1} \sum_{\lambda, \sigma_{1}, \sigma_{1}^{\prime}} \lambda \psi_{\sigma_{1} \lambda}^{* \sigma} \psi_{\sigma_{1}^{\prime} \lambda}^{\sigma^{\prime} \lambda}\right]
\end{aligned}
$$

Finally, the expectation value of the transverse spin operator is given by

$$
\left\langle\psi_{s^{1}}\left|M \mathcal{J}^{1}\right| \psi_{s^{1}}\right\rangle=\left\langle\psi_{s^{1}}\left|\frac{1}{2} F^{2} P^{+}+K^{3} P^{2}-\frac{1}{2} E^{2} P^{-}-P^{1} \mathcal{J}^{3}\right| \psi_{s^{1}}\right\rangle
$$

From the above expressions it is clear that all the explicit $P^{\perp}$ dependent terms get canceled in the final expression. To be specific, it can be easily seen that all the terms in the expectation value of $K^{3} P^{2}-\frac{1}{2} E^{2} P_{\text {free }}^{-}-P^{1} \mathcal{J}_{\text {orbital }}^{3}$ are $P^{\perp}$ dependent and they exactly cancel the $P^{\perp}$ dependent terms in $\frac{1}{2} F_{I(\text { free })}^{2} P^{+}$; the two $P^{1}$ dependent terms in the intrinsic part of $P^{1} \mathcal{J}^{3}$ exactly cancel the two similar terms in the expectation value of $\frac{1}{2} F_{I I}^{2} P^{+}+\frac{1}{2} F_{I I I}^{2} P^{+}$and the expectation value of $\frac{1}{2} E^{2} P_{\text {int }}^{-}$completely cancel all the $P^{\perp}$ dependent terms in the expectation value of $\frac{1}{2} F_{I(\text { int })}^{2} P^{+}$.

\section{APPENDIX E: DETAILS OF THE CALCULATION}

Here, we explicitly show the evaluation of one of the matrix elements of the interaction part of $F_{I}^{2}$. Consider the operator

$$
O_{g}=\frac{1}{2} \int d x^{-} d^{2} x^{\perp} x^{2} g\left[\frac{\left(\tilde{\sigma}^{\perp} \cdot \partial^{\perp}+m\right)}{\partial^{+}} \xi^{\dagger}\right]\left(\tilde{\sigma} \cdot A^{\perp}\right) \xi \frac{P^{+}}{2} .
$$

This can be written in Fock space as

$$
\begin{aligned}
O_{g}= & \frac{g}{2} \sum_{s_{1}, s_{2}, \lambda} \int\left(d k_{1}\right) \int\left(d k_{2}\right) \int\left[d k_{3}\right]\left(b^{\dagger}\left(k_{1}, s_{1}\right) a\left(k_{3}, \lambda\right) b\left(k_{2}, s_{2}\right) \chi_{s_{1}}^{\dagger} \frac{\left(\tilde{\sigma}^{\perp} \cdot k_{1}^{\perp}-i m\right)}{k_{1}^{+}}\left(\tilde{\sigma}^{\perp} \cdot \epsilon_{\lambda}^{\perp}\right) \chi_{s_{2}}\right. \\
& \times i \frac{\partial}{\partial k_{1}^{2}} 2(2 \pi)^{3} \delta^{3}\left(k_{1}-k_{2}-k_{3}\right)+b^{\dagger}\left(k_{1}, s_{1}\right) a^{\dagger}\left(k_{3}, \lambda\right) b\left(k_{2}, s_{2}\right) \chi_{s_{1}}^{\dagger} \frac{\left(\tilde{\sigma}^{\perp} \cdot k_{1}^{\perp}-i m\right)}{k_{1}^{+}}\left(\tilde{\sigma}^{\perp} \cdot \epsilon_{\lambda}^{* \perp}\right) \chi_{s_{2}} \\
& \left.\times i \frac{\partial}{\partial k_{1}^{2}} 2(2 \pi)^{3} \delta^{3}\left(k_{1}-k_{2}+k_{3}\right)\right) \frac{P^{+}}{2}
\end{aligned}
$$

where $(d k)=d k^{+} d^{2} k^{\perp} / 2(2 \pi)^{3} \sqrt{k}^{+}$and $[d k]=d k^{+} d^{2} k^{\perp} / 2(2 \pi)^{3} k^{+}$. We evaluate the expectation value of this operator for the dressed quark state given by Eq. (D1). Only the off-diagonal parts of the matrix element will give non-zero contribution. The matrix element is given by 


$$
\begin{aligned}
\left\langle\psi_{\sigma}\left|O_{g}\right| \psi_{\sigma^{\prime}}\right\rangle= & \frac{g}{2} \sum_{\sigma_{1}, \lambda} \int(d P)^{\prime} \int\left\{d p_{1}\right\} \int\left\{d p_{2}\right\} \sqrt{2(2 \pi)^{3}} P^{+} \delta^{3}\left(P-p_{1}-p_{2}\right) \\
& \times\left\{\phi_{1}^{* \sigma} \sqrt{p_{1}^{+}} \chi_{\sigma}^{\dagger} \frac{\left(\tilde{\sigma}^{\perp} \cdot P^{\perp}-i m\right)}{P^{+}}\left(\tilde{\sigma}^{\perp} \cdot \epsilon_{\lambda}^{\perp}\right) \chi_{\sigma_{1}} \phi_{\sigma_{1} \lambda^{\prime}}^{\sigma^{\prime}} i \frac{\partial}{\partial P^{2}} 2(2 \pi)^{3} \delta^{3}\left(P-p_{1}-p_{2}\right) \frac{1}{2} P^{+}+\text {H.c. }\right\} \\
= & -\frac{i g}{4} \int(d P)^{\prime} \int\left\{d p_{1}\right\} \int\left\{d p_{2}\right\} \sum_{\sigma_{1}, \lambda} \sqrt{2(2 \pi)^{3}} P^{+} \\
& \times\left[\phi_{1}^{* \sigma}\left[\frac{\partial}{\partial P^{2}} \phi_{\sigma_{1} \lambda}^{\sigma^{\prime}} \delta^{3}\left(P-p_{1}-p_{2}\right) \sqrt{p_{1}^{+}} \chi_{\sigma}^{\dagger} \frac{\left(\tilde{\sigma}^{\perp} \cdot P^{\perp}-i m\right)}{P^{+}}\left(\tilde{\sigma}^{\perp} \cdot \epsilon_{\lambda}^{\perp}\right) \chi_{\sigma^{\prime}}\right] 2(2 \pi)^{3} \delta^{3}\left(P-p_{1}-p_{2}\right) P^{+}+\text {H.c. }\right] \\
= & -\frac{i g}{4} \frac{1}{\sqrt{2(2 \pi)^{3}}} \sum_{\sigma_{1}, \lambda} \int(d P)^{\prime} \int d x d^{2} q^{\perp} \frac{1}{\sqrt{1-x}}\left[\psi_{1}^{* \sigma} \frac{\partial \psi_{\sigma_{1} \lambda}^{\sigma^{\prime}}}{\partial P^{2}} \chi_{\sigma}^{\dagger}\left(\tilde{\sigma}^{\perp} \cdot P^{\perp}-i m\right)\left(\tilde{\sigma}^{\perp} \cdot \epsilon_{\lambda}^{\perp}\right) \chi_{\sigma^{\prime}}+\text { H.c. }\right] \\
& -\frac{i g}{4} \frac{1}{\sqrt{2(2 \pi)^{3}}} \sum_{\sigma_{1}, \lambda} \int(d P)^{\prime} \int d x d^{2} q^{\perp} \frac{1}{\sqrt{1-x}}\left[\psi_{1}^{* \sigma} \psi_{\sigma_{1} \lambda}^{\sigma^{\prime}} \chi_{\sigma}^{\dagger} \tilde{\sigma}^{2}\left(\tilde{\sigma}^{\perp} \cdot \epsilon_{\lambda}^{\perp}\right) \chi_{\sigma^{\prime}}+\text { H.c. }\right]
\end{aligned}
$$

where $\{d p\}=d p^{+} d^{2} p^{\perp} / \sqrt{2(2 \pi)^{3} p^{+}}$and $(d P)^{\prime}=\frac{1}{2} d P^{+} d^{2} p^{\perp} 2(2 \pi)^{3} P^{+}$.

The other terms can also be evaluated in a similar method.

[1] V. de Alfaro, S. Fubini, G. Furlan, and G. Rossetti, Currents in Hadron Physics (North-Holland, Amsterdam, 1973).

[2] K. Bardakci and M. B. Halpern, Phys. Rev. 176, 1686 (1968); D. E. Soper, Ph.D. thesis, Stanford University, 1971, SLAC137; H. Leutwyler and J. Stern, Ann. Phys. (N.Y.) 112, 94 (1978).

[3] For attempts to incorporate interactions, see, for example, E. Eichten, F. Feinberg, and J. F. Willemsen, Phys. Rev. D 8, 1204 (1973); R. Carlitz and W. K. Tung, ibid. 13, 3446 (1976).

[4] H. J. Melosh, Phys. Rev. D 9, 1095 (1974).

[5] M. Brisudova and R. J. Perry, Phys. Rev. D 54, 6453 (1996).

[6] S. J. Brodsky, H. C. Pauli, and S. S. Pinsky, Phys. Rep. 301, 299 (1998).

[7] A. Harindranath and Rajen Kundu, Phys. Rev. D 59, 116013 (1999).

[8] S. J. Brodsky, D. S. Hwang, B. Q. Ma, and I. Schmidt, hep-th/0003082.

[9] A. Harindranath, Asmita Mukherjee, and Raghunath Ratabole,
Phys. Lett. B 476, 471 (2000).

[10] K. G. Wilson, T. S. Walhout, A. Harindranath, W. M. Zhang, R. J. Perry, and S. D. Glazek, Phys. Rev. D 49, 6720 (1994).

[11] See, for example, F. Gursey, in High Energy Physics, edited by C. DeWitt and R. Omnes (Gordon \& Breach Science Publishers, New York, 1965).

[12] B. Bakamjian and L. H. Thomas, Phys. Rev. 92, 1300 (1950); H. Osborn, ibid. 176, 1514 (1968).

[13] X. Ji, Phys. Rev. Lett. 78, 610 (1997).

[14] T. Maslowski and S. Glazek, hep-th/9906140.

[15] A. Harindranath, Asmita Mukherjee, and Raghunath Ratabole, hep-th/0004192.

[16] R. J. Perry, Phys. Lett. B 300, 8 (1993); A. Harindranath and W.-M. Zhang, Phys. Rev. D 48, 4903 (1993).

[17] A. Harindranath and W.-M. Zhang, Phys. Lett. B 408, 347 (1997).

[18] See, for example, M. Burkhardt and H. El-Khozondar, Phys. Rev. D 60, 054504 (1999). 